\title{
An Implicit Energy-Conservative 2D Fokker-Planck Algorithm
}

\section{Difference Scheme}

\author{
L. Chacón, * D. C. Barnes, † D. A. Knoll, † and G. H. Miley*
}

* Fusion Studies Laboratory, University of Illinois at Urbana-Champaign, 103 South Goodwin Avenue, Urbana, Illinois 61801; †X-PA, Los Alamos National Laboratory, MS B259, Los Alamos, New Mexico 87545;

$\ddagger X$-HM, Los Alamos National Laboratory, MS D413, Los Alamos, New Mexico 87545

E-mail: chacon@lanl.gov, dbarnes@lanl.gov, nol@lanl.gov,g-miley@uiuc.edu

Received March 11, 1999; revised October 9, 1999

Numerical energy conservation in Fokker-Planck problems requires the energy moment of the Fokker-Planck equation to cancel exactly. However, standard discretization techniques not only do not observe this requirement (thus precluding exact energy conservation), but they also demand very refined meshes to keep the energy error under control. In this paper, a new difference scheme for multidimensional Fokker-Planck problems that improves the numerical cancellation of the energy moment is proposed. Crucial to this new development is the reformulation of the friction term in the Fokker-Planck collision operator using Maxwell stress tensor formalism. As a result, the Fokker-Planck collision operator takes the form of a double divergence operating on a tensor, which is suitable for particle and energy conservative differencing. Numerical results show that the new discretization scheme improves the cancellation of the energy moment integral over standard approaches by at least an order of magnitude. (c) 2000 Academic Press

Key Words: plasma simulation; conservative discretization; energy-conservative Fokker-Planck.

\section{INTRODUCTION}

Plasma modeling in fusion devices generally falls into one of two categories: either the plasma can be regarded as being in local thermal equilibrium (LTE), and a fluid treatment is adequate; or the fluid treatment must be abandoned, either because the collision meanfree-path is comparable to the dimension of the system (giving large Knudsen numbers) or due to sources and/or sinks in velocity space that do not allow LTE to occur. The latter is the 
case, for instance, in mirror devices, where the loss cones (sinks) preclude the plasma from achieving thermal equilibrium, and in inertial electrostatic confinement fusion systems, where an ion source causes a non-Maxwellian plasma.

The analysis of non-local-thermal-equilibrium (NLTE) plasmas requires a kinetic treatment, whose accuracy and reliability depends on an adequate description of the collisions occurring in the system. In fusion plasmas, where small-angle Coulomb scattering is predominant due to a high mean particle energy, this description is provided by the FokkerPlanck collision term. The Fokker-Planck collision operator presents important intrinsic symmetries (such as the preservation of particles, momentum, and energy), and observes the $\mathrm{H}$-theorem, which states that the entropy of the system will increase in time, and the LTE solution-if such equilibrium is allowed-is the Maxwell-Boltzmann distribution (thus reverting to a fluid description in the LTE limit).

However, these properties may not hold in the numerical representation of the FokkerPlanck collision operator. Chang and Cooper showed [1] that exact particle conservation can be achieved numerically in problems with any geometry and dimensionality by a suitable discretization of the Fokker-Planck collision operator. In the same reference, they proposed an interpolation technique - effective only in spherical geometry - that preserves the positivity of the solution and favors the convergence to the LTE limit. Energy conservation in one-dimensional Fokker-Planck problems was first addressed by Epperlein [2]. He showed that numerical energy conservation is conceptually and practically possible in one-dimensional Fokker-Planck problems for any time step, provided that the energy moment of the Fokker-Planck collision operator cancels numerically. However, generalizing Epperlein's energy-conservative method for multiple dimensions in velocity space is nontrivial because the implicit time integration requires inverting a dense, non-symmetric Jacobian matrix.

Two steps are identified in the implementation of an implicit energy-conservative solver for the multidimensional Fokker-Planck equation, namely, (1) develop a suitable difference scheme to ensure the numerical cancellation of the energy moment of the Fokker-Planck collision operator, and (2) deal efficiently with the dense, non-symmetric algebraic problem that results from such formulation, with minimal storage and run-time requirements. The latter requirement has been successfully accomplished by the authors in Ref. [3], using multigrid-preconditioned Jacobian-free Newton-Krylov iterative methods. It is the objective of this paper to address the former for multidimensional geometries.

The paper is organized as follows. Section 2 deals with the theoretical background pertaining to the Fokker-Planck collision operator. The particle and energy conservative discretization method is derived in Section 3. The scaling of the energy error is discussed in Section 4. Section 5 presents some results to illustrate the performance and limitations of the new discretization technique vs the standard second-order particle-conservative discretization of the Fokker-Planck collision operator.

\section{THEORETICAL ISSUES OF ENERGY CONSERVATION IN THE FOKKER-PLANCK COLLISION OPERATOR}

The general form of the Boltzmann transport equation for a species $\alpha$ reads

$$
\frac{\partial f_{\alpha}}{\partial t}+\mathbf{v} \cdot \frac{\partial f_{\alpha}}{\partial \mathbf{r}}+\frac{\mathbf{F}_{\alpha}}{m_{\alpha}} \cdot \frac{\partial f_{\alpha}}{\partial \mathbf{v}}=\sum_{\beta} L_{\alpha \beta}\left(f_{\alpha}, f_{\beta}\right)
$$


where $f_{\alpha}$ is the distribution function of the species $\alpha, \mathbf{F}_{\alpha}$ is the force, $m_{\alpha}$ is the mass, $L_{\alpha \beta}$ represents the effects of the species $\beta$ on the species $\alpha$ via collisions, and the sum is over all the species present in the system. In this equation (and in what follows), $t$ is time, $\mathbf{r}$ is the position vector, and $\mathbf{v}$ is the velocity vector. For a single species system in which Coulomb collisions are dominant, the collision term in the transport equation will be given by the Fokker-Planck collision operator, which in the Rosenbluth form [4] reads

$$
L_{\alpha \alpha}=-\Gamma_{\alpha} \frac{\partial}{\partial \mathbf{v}} \cdot\left[f_{\alpha} \frac{\partial H\left(f_{\alpha}\right)}{\partial \mathbf{v}}-\frac{1}{2} \frac{\partial}{\partial \mathbf{v}} \cdot\left(\frac{\partial^{2} G\left(f_{\alpha}\right)}{\partial \mathbf{v} \partial \mathbf{v}} f_{\alpha}\right)\right] .
$$

Here $\Gamma_{\alpha}=4 \pi e_{\alpha}^{4} \lambda / m_{\alpha}^{2}$, where $\lambda$ is the Coulomb logarithm, and $e_{\alpha}, m_{\alpha}$ are the charge and mass of the species $\alpha$. As is clear from Eq. (1), $L_{\alpha \alpha}$ operates only in velocity space. Hence, for the purpose of studying the energy conservation issues of the Fokker-Planck collision operator, the convection and field transport terms present in the most general form of the Boltzmann transport equation can be ignored (equivalent to assuming that the plasma is field-free and spatially homogeneous). This results in the following simplified FokkerPlanck equation,

$$
\frac{\partial f}{\partial t}=L(f)=-\Gamma \frac{\partial}{\partial \mathbf{v}} \cdot\left[f \frac{\partial H(f)}{\partial \mathbf{v}}-\frac{1}{2} \frac{\partial}{\partial \mathbf{v}} \cdot\left(\frac{\partial^{2} G(f)}{\partial \mathbf{v} \partial \mathbf{v}} f\right)\right]=-\frac{\partial}{\partial \mathbf{v}} \cdot \mathbf{J}_{F P},
$$

where the species subscript has been dropped for simplicity. In Eq. (2), $\mathbf{J}_{F P}$ is the FokkerPlanck flux, defined as

$$
\mathbf{J}_{F P}=\Gamma\left[f \frac{\partial H(f)}{\partial \mathbf{v}}-\frac{1}{2} \frac{\partial}{\partial \mathbf{v}} \cdot\left(\frac{\partial^{2} G(f)}{\partial \mathbf{v} \partial \mathbf{v}} f\right)\right]
$$

which is formed by a friction term (proportional to $f$ ) and a diffusion term (proportional to $\partial f / \partial \mathbf{v})$. The friction and diffusion coefficients are expressed in terms of $H(f)$ and $G(f)$, respectively, defined by

$$
\begin{aligned}
& \nabla_{\mathbf{v}}^{2} H=-8 \pi f \\
& \nabla_{\mathbf{v}}^{2} G=H .
\end{aligned}
$$

These are called the Rosenbluth potentials [4], due to the obvious similarity with electrostatic potential theory.

Equation (2) can be shown [5] to satisfy important intrinsic symmetries such as particle, momentum, and energy conservation, and preservation of the positivity of the solution. It also satisfies the H-theorem, which implies that the Maxwell-Boltzmann distribution is the solution in equilibrium. The preservation of these symmetries in the numerical approximation to the problem is essential to adequately simulate the physics. Particle conservation is straightforward to prove by virtue of Gauss' theorem,

$$
\frac{\partial N}{\partial t}=\int_{\Omega_{\infty}} d \mathbf{v} \frac{\partial f}{\partial t}=-\int_{\Omega_{\infty}} d \mathbf{v} \frac{\partial}{\partial \mathbf{v}} \cdot \mathbf{J}_{F P}=-\oint_{\partial \Omega_{\infty}} d \mathbf{S} \cdot \mathbf{J}_{F P}=0
$$

provided that $f, G$, and $H$ are regular at infinity. In this equation, $\Omega_{\infty}$ represents the infinite velocity domain, and $\partial \Omega_{\infty}$ is the boundary of that domain at infinity. The conservative 
discretization of the Fokker-Planck collision operator [1] results in a local particle balance at every node, thus ensuring particle conservation numerically with the proper boundary conditions. However, energy conservation is considerably more involved. It requires a global balance in an infinite velocity domain,

$$
\frac{\partial E}{\partial t}=\int_{\Omega_{\infty}} d \mathbf{v} \frac{v^{2}}{2} \frac{\partial f}{\partial t}=\int_{\Omega_{\infty}} d \mathbf{v} \frac{v^{2}}{2} L(f)=-\int_{\Omega_{\infty}} d \mathbf{v} \frac{v^{2}}{2} \frac{\partial}{\partial \mathbf{v}} \cdot \mathbf{J}_{F P}(f)=0 .
$$

Energy conservation cannot be shown from Eqs. (2) and (6) directly unless the Rosenbluth potentials are expressed as Poisson integrals (leading to the Landau formalism [5]). Calculating these integrals is computationally much more expensive than inverting the laplacian operators in $2 \mathrm{D}$. In fact, if $N$ is the total number of mesh points (equal to the total number of unknowns), calculating the Rosenbluth's potentials from the Poisson integral in a 2D velocity space would require $O\left(N^{2}\right)$ operations $(O(N)$ operations per integral per mesh point), whereas solving Poisson's equation numerically would require $O\left(N^{3 / 2}\right)$ with the optimized successive over-relaxation method, and far less if preconditioned Krylov iterative techniques are employed [6]. Therefore, the Fokker-Planck equation has to be re-cast so that energy conservation can be demonstrated from the more efficient laplacian formulation of the Rosenbluth potentials. This will be accomplished here by using an analogy of the Maxwell stress tensor formalism in electromagnetic theory.

\subsection{Reformulation of the Fokker-Planck Equation}

The electrostatic analogy introduced by Rosenbluth suggests that $H$ can be regarded as a potential, $\partial H / \partial \mathbf{v}$ as the corresponding electric field, and $2 f$ as the charge density. Then, we can write

$$
\frac{\partial H}{\partial \mathbf{v}} f=-\frac{1}{8 \pi} \frac{\partial}{\partial \mathbf{v}} \cdot\left[\frac{\partial H}{\partial \mathbf{v}} \frac{\partial H}{\partial \mathbf{v}}-\frac{\bar{I}}{2}\left(\frac{\partial H}{\partial \mathbf{v}}\right)^{2}\right]=-\frac{\partial}{\partial \mathbf{v}} \cdot \bar{T}[H, H],
$$

where $\bar{I}$ is the identity dyadic, and $\bar{T}[H, H]$ is formally equivalent to the Maxwell stress tensor [7]. This tensor is a symmetric, bilinear operator. Using it, Eq. (2) transforms into

$$
\frac{\partial f}{\partial t}=\Gamma \frac{\partial}{\partial \mathbf{v}} \cdot \frac{\partial}{\partial \mathbf{v}} \cdot\left[\bar{T}[H, H]+\frac{1}{2} \frac{\partial^{2} G(f)}{\partial \mathbf{v} \partial \mathbf{v}} f\right] .
$$

Note that $f$ no longer appears in the friction term of the Fokker-Planck equation, which is represented now by the divergence of the tensor $\bar{T}[H, H]$. This new formulation of the Fokker-Planck collision operator, developed to demonstrate energy conservation within the Rosenbluth potentials formalism, is subsequently referred to as the tensor Fokker-Planck formalism.

\subsection{Energy Conservation in the Tensor Fokker-Planck Formalism}

The rate of change of energy in an ensemble of particles distributed according to $f$ in an infinite velocity domain $\Omega_{\infty}$ is given by

$$
\frac{\partial E}{\partial t}=\int_{\Omega_{\infty}} d \mathbf{v} \frac{v^{2}}{2} \frac{\partial f}{\partial t}
$$


which, after introducing Eq. (8) and integrating by parts once, reads as

$$
\begin{aligned}
\frac{\partial E}{\partial t} & =-\int_{\Omega_{\infty}} d \mathbf{v} \frac{v^{2}}{2} \frac{\partial}{\partial \mathbf{v}} \cdot \mathbf{J}_{F P}=\int_{\Omega_{\infty}} d \mathbf{v} \mathbf{v} \cdot \mathbf{J}_{F P} \\
& =-\Gamma\left\{\int_{\Omega_{\infty}} d \mathbf{v} \mathbf{v} \cdot \frac{\partial}{\partial \mathbf{v}} \cdot \bar{T}[H, H]+\frac{1}{2} \int_{\Omega_{\infty}} d \mathbf{v} \mathbf{v} \cdot \frac{\partial}{\partial \mathbf{v}} \cdot\left[\frac{\partial^{2} G(f)}{\partial \mathbf{v} \partial \mathbf{v}} f\right]\right\} .
\end{aligned}
$$

In what follows, the proportionality constant $\Gamma$ is set to one for simplicity. The boundary integrals, which are zero at infinity, are omitted here. The second integral in Eq. (10) is integrated again by parts to find, according to the definitions of $H$ and $G$,

$$
\int_{\Omega_{\infty}} d \mathbf{v} \mathbf{v} \cdot \frac{\partial}{\partial \mathbf{v}} \cdot\left[\frac{\partial^{2} G(f)}{\partial \mathbf{v} \partial \mathbf{v}} f\right]=-\int_{\Omega_{\infty}} d \mathbf{v} f \nabla_{\mathbf{v}}^{2} G(f)=\frac{1}{8 \pi} \int_{\Omega_{\infty}} d \mathbf{v} H \nabla_{\mathbf{v}}^{2} H .
$$

At this point, the bilinear, symmetric operator $Q[H, H]$ is defined as

$$
Q[H, H]=\frac{H \nabla_{\mathbf{v}}^{2} H}{16 \pi}=\frac{1}{16 \pi}\left[\frac{\partial^{2}}{\partial \mathbf{v}^{2}}\left(\frac{H^{2}}{2}\right)-\left(\frac{\partial H}{\partial \mathbf{v}}\right)^{2}\right]
$$

leading to the following expression for the energy rate of change:

$$
\frac{\partial E}{\partial t}=-\int_{\Omega_{\infty}} d \mathbf{v}\left[\mathbf{v} \cdot \frac{\partial}{\partial \mathbf{v}} \cdot \bar{T}[H, H]+Q[H, H]\right]=0 .
$$

The rate of change of energy cancels because

$$
\begin{aligned}
\int_{\Omega_{\infty}} d \mathbf{v} \mathbf{v} \cdot \frac{\partial}{\partial \mathbf{v}} \cdot \bar{T}[H, H] & =\frac{1}{16 \pi} \int_{\Omega_{\infty}} d \mathbf{v}\left(\frac{\partial H}{\partial \mathbf{v}}\right)^{2} \\
\int_{\Omega_{\infty}} d \mathbf{v} Q[H, H] & =-\frac{1}{16 \pi} \int_{\Omega_{\infty}} d \mathbf{v}\left(\frac{\partial H}{\partial \mathbf{v}}\right)^{2} .
\end{aligned}
$$

The previous result has been obtained for an infinite velocity domain $\Omega_{\infty}$, but it also holds for finite domains $\Omega$, limited by a surface boundary $\partial \Omega$, provided that $f$ is absolutely contained in $\Omega$. This is proven next. For a finite domain, the integrations in Eqs. (14) and (15) yield

$$
\begin{aligned}
\int_{\Omega} d \mathbf{v} \mathbf{v} \cdot \frac{\partial}{\partial \mathbf{v}} \cdot \bar{T}[H, H]= & \frac{1}{16 \pi} \int_{\Omega} d \mathbf{v}\left(\frac{\partial H}{\partial \mathbf{v}}\right)^{2}+\frac{1}{8 \pi} \oint_{\partial \Omega} d \mathbf{S} \\
& \cdot\left[\frac{\partial H}{\partial \mathbf{v}}\left(\mathbf{v} \cdot \frac{\partial H}{\partial \mathbf{v}}\right)-\frac{\mathbf{v}}{2}\left(\frac{\partial H}{\partial \mathbf{v}}\right)^{2}\right] \\
\int_{\Omega} d \mathbf{v} Q[H, H]= & -\frac{1}{16 \pi} \int_{\Omega} d \mathbf{v}\left(\frac{\partial H}{\partial \mathbf{v}}\right)^{2}+\frac{1}{16 \pi} \oint_{\partial \Omega} d \mathbf{S} \cdot \frac{\partial H}{\partial \mathbf{v}} H
\end{aligned}
$$

and the corresponding energy rate of change reads

$$
\frac{\partial E}{\partial t} \propto \underbrace{\oint_{\partial \Omega} d \mathbf{S} \cdot \frac{1}{2} \mathbf{v}\left(\frac{\partial H}{\partial \mathbf{v}}\right)^{2}}_{I_{1}}-\underbrace{\oint_{\partial \Omega} d \mathbf{S} \cdot \frac{\partial H}{\partial \mathbf{v}}\left(\mathbf{v} \cdot \frac{\partial H}{\partial \mathbf{v}}+\frac{H}{2}\right)}_{I_{2}} .
$$


Using Gauss' integral theorem, the integral $I_{2}$ is transformed to a volume integral over the volume in velocity space external to $\Omega$ (represented by $\Omega_{\text {ext }}$ ), also limited by $\partial \Omega$ and extending to infinity (i.e., $\Omega \cup \Omega_{\text {ext }}=\Omega_{\infty}$ ). Then, Eq. (18) transforms into

$$
\frac{\partial E}{\partial t} \propto \underbrace{\oint_{\partial \Omega} d \mathbf{S} \cdot \frac{1}{2} \mathbf{v}\left(\frac{\partial H}{\partial \mathbf{v}}\right)^{2}}_{I_{1}}+\underbrace{\int_{\Omega_{e x t}} d \mathbf{v} \frac{\partial}{\partial \mathbf{v}} \cdot\left[\frac{\partial H}{\partial \mathbf{v}}\left(\mathbf{v} \cdot \frac{\partial H}{\partial \mathbf{v}}+\frac{H}{2}\right)\right]}_{I_{2}} .
$$

Note that the sign in the integral $I_{2}$ has changed because $d \mathbf{S}=-d \mathbf{S}_{\text {ext }}$. As $f$ is zero outside $\Omega, H$ is harmonic in $\Omega_{\text {ext }}$, and $I_{2}$ simplifies to

$$
\begin{aligned}
I_{2} & =\int_{\Omega_{e x t}} d \mathbf{v} \frac{\partial}{\partial \mathbf{v}} \cdot\left[\frac{\partial H}{\partial \mathbf{v}}\left(\mathbf{v} \cdot \frac{\partial H}{\partial \mathbf{v}}+\frac{H}{2}\right)\right]=\int_{\Omega_{e x t}} d \mathbf{v}\left[\frac{3}{2}\left(\frac{\partial H}{\partial \mathbf{v}}\right)^{2}+\frac{\partial H}{\partial \mathbf{v}} \cdot \frac{\partial^{2} H}{\partial \mathbf{v} \partial \mathbf{v}} \cdot \mathbf{v}\right] \\
& =\frac{1}{2} \int_{\Omega_{e x t}} d \mathbf{v} \frac{\partial}{\partial \mathbf{v}} \cdot\left[\mathbf{v}\left(\frac{\partial H}{\partial \mathbf{v}}\right)^{2}\right]=-\oint_{\partial \Omega} d \mathbf{S} \cdot \frac{1}{2} \mathbf{v}\left(\frac{\partial H}{\partial \mathbf{v}}\right)^{2} .
\end{aligned}
$$

Therefore

$$
\frac{\partial E}{\partial t} \propto \oint_{\partial \Omega} d \mathbf{S} \cdot \frac{1}{2} \mathbf{v}\left(\frac{\partial H}{\partial \mathbf{v}}\right)^{2}-\oint_{\partial \Omega} d \mathbf{S} \cdot \frac{1}{2} \mathbf{v}\left(\frac{\partial H}{\partial \mathbf{v}}\right)^{2}=0 .
$$

Hence, the energy moment also cancels in finite domains provided that $f$ is absolutely confined in them. The importance of this result stems from the fact that only finite domains can be considered in numerical problems. However, exact numerical cancellation will not occur due to numerical errors in the boundary integrals above. This is in fact the weakness of any energy-conservative discretization technique in Fokker-Planck problems. A detailed analysis of the errors in this cancellation is presented in Section 4.

\section{DISCRETIZATION OF THE FOKKER-PLANCK COLLISION OPERATOR}

The simplified transport equation in Eq. (2) represents the time evolution of an ensemble of particles in velocity space. Then, in the numerical description of the problem, two distinct steps are identified, namely, the discretization in time and the discretization in velocity space. Both steps are crucial for the adequate preservation of particles and energy - as well as the accuracy and consistency of the numerical solution. In this paper we deal mainly with the velocity space aspect of the problem. The details of the time integration of the non-linear equation, and the solution algorithm used on the resulting linear system, are described in detail in Ref. [3].

For the energy-conservative time discretization to hold when the Fokker-Planck collision operator $L(f)$ is discretized in velocity space, the identity

$$
\frac{\partial E}{\partial t}=\int_{\Omega_{\infty}} d \mathbf{v} \frac{v^{2}}{2} \frac{\partial f}{\partial t}=-\Gamma \int_{\Omega_{\infty}} d \mathbf{v}\left[\mathbf{v} \cdot \frac{\partial}{\partial \mathbf{v}} \cdot \bar{T}[H, H]+Q[H, H]\right]=0
$$

must be satisfied numerically. The next sections describe a systematic way of designing a discretization technique that accomplishes this goal. The process, which follows the integration order followed in obtaining Eq. (13) (reproduced in Eq. (21)), renders, as a 


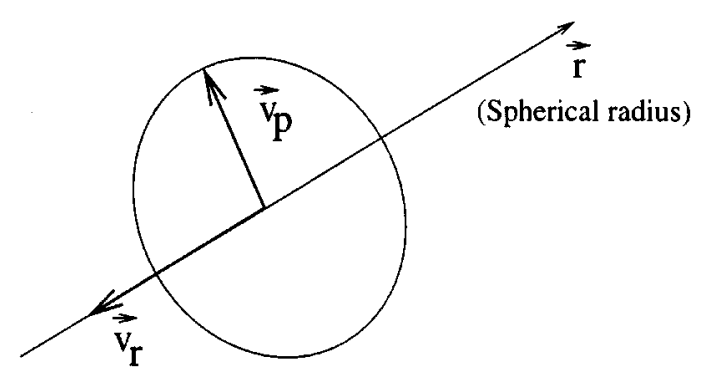

FIG. 1. Diagram of the local cylindrical velocity coordinate system $\left(\mathbf{v}_{r}, \mathbf{v}_{p}\right)$ considered in this work. Cylindrical symmetry is assumed. The spherical radius vector $\mathbf{r}$ is included for reference.

by-product, the required numerical representation of the subordinate problems for $H$ and $G$, which will be somewhat different from the traditional treatment.

\subsection{Definition of the Computational Velocity Domain}

So far, the discussion has been independent of a particular geometry and/or dimensionality in velocity space. To focus the discussion that follows, a 2D cylindrical velocity space with angular symmetry is adopted. This space is spanned by $\left(v_{r}, v_{p}\right)$, where $v_{r}$ is the cylindrical $z$-axis, and $v_{p}$ is the cylindrical $r$-axis (Fig. 1 ), and $v_{r} \in\left[0, v_{\text {limit }}\right] ; v_{p} \in\left[0, v_{\text {limit }}\right]$. Here, $v_{\text {limit }}$ is typically set to several times the characteristics velocity of the problem, $v_{0}$.

The domain is discretized with an integer mesh and a half mesh (Fig. 2). The integer mesh is defined using $N_{r}(+1)$ nodes in the $v_{r}$ axis, and $N_{p}(+1)$ nodes in the $v_{p}$ axis, with the constraints

$$
\begin{aligned}
v_{r, 1}=0, & v_{r, N_{r}}=v_{\text {limit }} \\
v_{p, 1}=0, & v_{p, N_{p}}=v_{\text {limit }} .
\end{aligned}
$$

Each velocity node is characterized by a pair $\left(v_{r, i}, v_{p, j}\right)$, with $i=1, \ldots, N_{r}(+1)$, and $j=1, \ldots, N_{p}(+1)$. The additional $\left(i=N_{r}+1, j\right)$ and $\left(i, j=N_{p}+1\right)$ nodes at the boundaries will serve a double purpose: (1) they will be used to impose the far-field boundary conditions for the Rosenbluth potentials, and (2) they will allow an accurate determination of the friction and diffusion coefficients of the Fokker-Planck collision operator at the

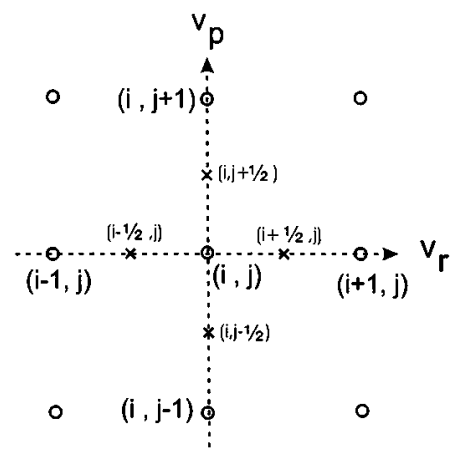

FIG. 2. Diagram of the 9-point stencil in velocity space employed in the discretization of the Fokker-Planck collision operator. 
outer boundaries. These coefficients are defined as first and second order derivatives of the Rosenbluth potentials, and hence require at least two neighboring nodes to be estimated accurately. This will turn out to be essential, because errors at the boundaries will ultimately lead to errors in the conservation of energy.

In addition to the integer mesh, a half mesh is introduced (Fig. 2) to assist in the development of an energy-conservative discretization, defined as

$$
\begin{aligned}
v_{r, i+\frac{1}{2}}=\frac{v_{r, i+1}+v_{r, i}}{2} ; & i=1, \ldots, N_{r} \\
v_{p, j+\frac{1}{2}}=\frac{v_{p, j+1}+v_{p, j}}{2} ; & j=1, \ldots, N_{p} .
\end{aligned}
$$

The velocity increments in the integer and half mesh (which will be non-uniform in general) are obtained as

$$
\begin{array}{rlrl}
\Delta v_{r, i} & =v_{r, i+\frac{1}{2}}-v_{r, i-\frac{1}{2}} ; & i & =1, \ldots, N_{r} \\
\Delta v_{p, j} & =v_{p, j+\frac{1}{2}}-v_{p, j-\frac{1}{2}} ; & j & =1, \ldots, N_{p} \\
\Delta v_{r, i+\frac{1}{2}} & =v_{r, i+1}-v_{r, i} ; & i & =1, \ldots, N_{r} \\
\Delta v_{p, j+\frac{1}{2}} & =v_{p, j+1}-v_{p, j} ; \quad j & j=1, \ldots, N_{p} .
\end{array}
$$

Finally, it will be useful to define an additional mesh in the perpendicular direction,

$$
v_{p, j}^{*}=\frac{v_{p, j+\frac{1}{2}}+v_{p, j-\frac{1}{2}}}{2} ; \quad v_{p, 1}^{*}=0 ; j=2, \ldots, N_{p}
$$

and $v_{p, 1}^{*}=0$. Note that, for a uniform mesh, $v_{p, j}^{*}=v_{p, j}$.

\subsection{Boundary Conditions in Velocity Space}

The computational domain defined in the previous section is limited by four distinct boundaries, namely, $v_{r}=0, v_{p}=0$ (inner boundaries), and $v_{r}=v_{\text {limit }}, v_{p}=v_{\text {limit }}$ (outer boundaries). The system is assumed symmetric with respect to both inner boundaries; hence

$$
\begin{aligned}
& \left.\frac{\partial}{\partial v_{p}}\right|_{v_{p}=0}=0 \\
& \left.\frac{\partial}{\partial v_{r}}\right|_{v_{r}=0}=0 .
\end{aligned}
$$

Regarding the outer boundaries, the conditions are different for the main Fokker-Planck problem $(f)$ and the two subordinate Poisson problems $(H, G)$. These are described next.

3.2.1. Boundary conditions for the Rosenbluth potentials. The approximate numerical cancellation of the boundary integrals in Eq. (20) requires that $H$ (and $G$, by consistency) be determined in the finite domain $\Omega$ and at the boundary as if they actually extended to infinity. This is accomplished by integrating the Rosenbluth potentials with the so-called far-field boundary conditions [7], obtained by calculating $H$ and $G$ at the nodes of the outer 
boundaries from the integral definition of the Rosenbluth potentials, based on the Green's function formalism for an infinite domain:

$$
H(\mathbf{v})=2 \int_{\Omega_{\infty}} d \mathbf{v}^{\prime} \frac{f\left(\mathbf{v}^{\prime}\right)}{\left|\mathbf{v}-\mathbf{v}^{\prime}\right|} ; \quad G(\mathbf{v})=\int_{\Omega_{\infty}} d \mathbf{v}^{\prime} f\left(\mathbf{v}^{\prime}\right)\left|\mathbf{v}-\mathbf{v}^{\prime}\right| .
$$

For the particular case of a cylindrical coordinate system, these integrals can be expanded in terms of complete elliptic integrals of the first kind $K(k)$ and second kind $E(k)$ as

$$
\begin{aligned}
H\left(v_{r}, v_{p}\right)= & 8 \int_{0}^{v_{\text {limit }}} v_{p}^{\prime} d v_{p}^{\prime} \int_{0}^{v_{\text {limit }}} d v_{r}^{\prime} \frac{f\left(v_{r}^{\prime}, v_{p}^{\prime}\right) K\left[k\left(v_{r}, v_{p} ; v_{r}^{\prime}, v_{p}^{\prime}\right)\right]}{\sqrt{\left(v_{p}+v_{p}^{\prime}\right)^{2}+\left(v_{r}-v_{r}^{\prime}\right)^{2}}} \\
G\left(v_{r}, v_{p}\right)= & 4 \int_{0}^{v_{\text {limit }}} v_{p}^{\prime} d v_{p}^{\prime} \int_{0}^{v_{\text {limit }}} d v_{r}^{\prime} f\left(v_{r}^{\prime}, v_{p}^{\prime}\right) E\left[k\left(v_{r}, v_{p} ; v_{r}^{\prime}, v_{p}^{\prime}\right)\right] \\
& \times \sqrt{\left(v_{p}+v_{p}^{\prime}\right)^{2}+\left(v_{r}-v_{r}^{\prime}\right)^{2}},
\end{aligned}
$$

where $k\left(v_{r}, v_{p} ; v_{r}^{\prime}, v_{p}^{\prime}\right)=4 v_{p} v_{p}^{\prime} / \sqrt{\left(v_{p}+v_{p}^{\prime}\right)^{2}+\left(v_{r}-v_{r}^{\prime}\right)^{2}}$. The functions $K(k)$ and $E(k)$ are calculated numerically with well-known polynomial approximations [8]. Note that the computational complexity associated with the calculation of the far-field boundary conditions is $O\left(N^{3 / 2}\right)$ (the computational complexity of the integrals is $O(N)$, and there are $O(\sqrt{N})$ boundary points).

3.2.2. Boundary conditions for the Fokker-Planck equation. The boundary conditions at the outer boundaries in the Fokker-Planck problem are selected so that particles are conserved exactly. This issue is discussed further in the next section.

\subsection{Particle-Conservative Discretization of the Fokker-Planck Collision Operator}

The first step in finding the discrete Fokker-Planck collision operator, $\Lambda_{i, j}\left(f_{l, m}\right)$, from $L(f)=-(\partial / \partial \mathbf{v}) \cdot \mathbf{J}_{F P}$ is to discretize the divergence operator conservatively. Here, $\mathbf{J}_{F P}$ is the Fokker-Planck flux, given by

$$
\mathbf{J}_{F P}=-\Gamma \frac{\partial}{\partial \mathbf{v}} \cdot\left[\bar{T}[H, H]+\frac{1}{2} \frac{\partial^{2} G(f)}{\partial \mathbf{v} \partial \mathbf{v}} f\right] .
$$

In a cylindrical space with angular symmetry, $\mathbf{J}_{F P}=R \mathbf{u}_{p}+Z \mathbf{u}_{r}$ (where $\mathbf{u}_{p}=\mathbf{v}_{p} / v_{p}$ is the unit vector in the $v_{p}$-direction, and $\mathbf{u}_{r}=\mathbf{v}_{r} / v_{r}$ is the unit vector in the $v_{r}$-direction). Then, the divergence operator operating on $\mathbf{J}_{F P}$ reads

$$
\frac{\partial}{\partial \mathbf{v}} \cdot \mathbf{J}_{F P}=\frac{1}{v_{p}} \frac{\partial}{\partial v_{p}}\left(v_{p} R\right)+\frac{\partial Z}{\partial v_{r}} .
$$

However, to avoid the singularity at $v_{p}=0$, the integral form of the divergence operator will be used instead to discretize the divergence of the Fokker-Planck flux,

$$
\int_{\Delta \Omega_{i, j}} d \mathbf{v} \frac{\partial}{\partial \mathbf{v}} \cdot \mathbf{J}_{F P}=\oint_{\partial \Omega_{i, j}} d \mathbf{S} \cdot \mathbf{J}_{F P}
$$

where $d \mathbf{S}$ is a vector normal to the surface and pointing outwards from the volume, and $\Delta \Omega_{i, j}$ is the cylindrical control volume associated with a velocity node $(i, j)$ (Fig. 3 ). 


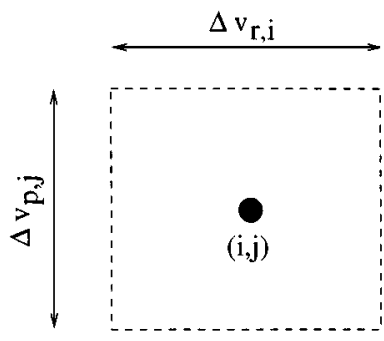

Discretization control volume

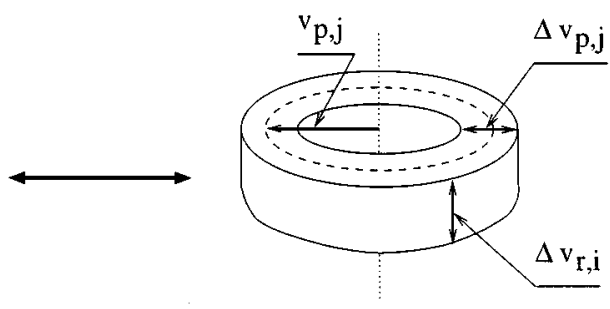

Physical volume

FIG. 3. Correspondence between the discretization control volume and the physical volume in velocity space. This is useful to define the volume elements $\left(\Delta \Omega_{i, j}\right)$ and the surface elements $\left(\Delta S_{i \pm 1 / 2, j}, \Delta S_{i, j \pm 1 / 2}\right)$, present in the discretized equations.

Assuming this volume is sufficiently small, Eq. (32) can be approximated by the following particle flux balance,

$$
\begin{aligned}
\Delta \Omega_{i, j}\left[\frac{\partial}{\partial \mathbf{v}} \cdot \mathbf{J}_{F P}\right]_{i, j}= & \Delta S_{i+\frac{1}{2}, j} Z_{i+\frac{1}{2}, j}-\Delta S_{i-\frac{1}{2}, j} Z_{i-\frac{1}{2}, j} \\
& +\Delta S_{i, j+\frac{1}{2}} R_{i, j+\frac{1}{2}}-\Delta S_{i, j-\frac{1}{2}} R_{i, j-\frac{1}{2}},
\end{aligned}
$$

where the $\Delta S$ 's are the areas of the boundaries of the volume element $\Delta \Omega_{i, j}$. Hence

$$
\begin{aligned}
-\Lambda_{i, j}(f) & =\left[\frac{\partial}{\partial \mathbf{v}} \cdot \mathbf{J}_{F P}\right]_{i, j} \\
& =\frac{\Delta S_{i+\frac{1}{2}, j} Z_{i+\frac{1}{2}, j}-\Delta S_{i-\frac{1}{2}, j} Z_{i-\frac{1}{2}, j}}{\Delta \Omega_{i, j}}+\frac{\Delta S_{i, j+\frac{1}{2}} R_{i, j+\frac{1}{2}}-\Delta S_{i, j-\frac{1}{2}} R_{i, j-\frac{1}{2}}}{\Delta \Omega_{i, j}} .
\end{aligned}
$$

The appropriate expressions for $\Delta S_{i \pm \frac{1}{2}, j}, \Delta S_{i, j \pm \frac{1}{2}}$, and $\Delta \Omega_{i, j}$ will depend on the location of the node $(i, j)$ in the mesh. In addition, the particle-conservative boundary conditions must be enforced at the boundaries, which will result in one of the components of the flux being zero. Details are given in Appendix A.

\subsection{Energy-Conservative Discretization of the Fokker-Planck Collision Operator}

The discretization in Eq. (34) will be the starting point for the development of the energy-conservative discretization scheme. Such development will consist in tailoring the discretization of the flux components, $R_{i, j \pm \frac{1}{2}}$ and $Z_{i \pm \frac{1}{2}, j}$ (not uniquely defined at this point) adequately, by following the path indicated by the theoretical proof presented in Subsection 2.2.

3.4.1. Numerical integration by parts of the energy moment. Before attempting the discretization of the flux components, it is essential to show that the discretized FokkerPlanck operator satisfies a numerical equivalent of the following integration by parts (over which rests the theoretical proof of energy conservation, as shown in Eq. (10)):

$$
\int d \mathbf{v} \frac{v^{2}}{2} \frac{\partial}{\partial \mathbf{v}} \cdot \mathbf{J}_{F P}=-\int d \mathbf{v} \mathbf{v} \cdot \mathbf{J}_{F P}
$$


Discretizing the energy moment integral as the particle moment integral (Eqs. (32) and (33)), we find

$$
\begin{aligned}
& \int d \mathbf{v} \frac{v^{2}}{2} \frac{\partial}{\partial \mathbf{v}} \cdot \mathbf{J}_{F P} \\
& \approx \sum_{i, j} \Delta \Omega_{i, j} \frac{v_{i, j}^{2}}{2}\left[\frac{\partial}{\partial \mathbf{v}} \cdot \mathbf{J}_{F P}\right]_{i, j} \\
& =\sum_{i, j} \Delta \Omega_{i, j} \frac{v_{i, j}^{2}}{2}\left[\frac{\Delta S_{i+\frac{1}{2}, j} Z_{i+\frac{1}{2}, j}-\Delta S_{i-\frac{1}{2}, j} Z_{i-\frac{1}{2}, j}}{\Delta \Omega_{i, j}}+\frac{\Delta S_{i, j+\frac{1}{2}} R_{i, j+\frac{1}{2}}-\Delta S_{i, j-\frac{1}{2}} R_{i, j-\frac{1}{2}}}{\Delta \Omega_{i, j}}\right] \\
& =\sum_{i, j} \frac{v_{i, j}^{2}}{2}\left[\Delta S_{i+\frac{1}{2}, j} Z_{i+\frac{1}{2}, j}-\Delta S_{i-\frac{1}{2}, j} Z_{i-\frac{1}{2}, j}+\Delta S_{i, j+\frac{1}{2}} R_{i, j+\frac{1}{2}}-\Delta S_{i, j-\frac{1}{2}} R_{i, j-\frac{1}{2}}\right] .
\end{aligned}
$$

Factoring out the $(\Delta S R)$ and ( $\Delta S Z)$ terms (called "telescoping" the sum), results in

$$
\begin{aligned}
& \sum_{i, j} \Delta \Omega_{i, j} \frac{v_{i, j}^{2}}{2}\left[\frac{\partial}{\partial \mathbf{v}} \cdot \mathbf{J}_{F P}\right]_{i, j} \\
& \quad=\sum_{i, j}\left[\Delta S_{i, j+\frac{1}{2}} R_{i, j+\frac{1}{2}} \frac{v_{i, j}^{2}-v_{i, j+1}^{2}}{2}+\Delta S_{i+\frac{1}{2}, j} Z_{i+\frac{1}{2}, j} \frac{v_{i, j}^{2}-v_{i+1, j}^{2}}{2}\right] .
\end{aligned}
$$

In general, this procedure fails at the boundaries, because the boundary terms cannot be "paired." However, the flux components at the boundaries are identically zero-as required by particle conservation-and the expression above is exact.

According to Eqs. (22), (23), (26), and (27), we can write

$$
\begin{aligned}
& v_{i, j}^{2}-v_{i, j+1}^{2}=v_{p, j}^{2}-v_{p, j+1}^{2}=\left(v_{p, j}+v_{p, j+1}\right)\left(v_{p, j}-v_{p, j+1}\right)=-2 v_{p, j+\frac{1}{2}} \Delta v_{p, j+\frac{1}{2}} \\
& v_{i, j}^{2}-v_{i+1, j}^{2}=v_{r, i}^{2}-v_{r, i+1}^{2}=\left(v_{r, i}+v_{r, i+1}\right)\left(v_{r, i}-v_{r, i+1}\right)=-2 v_{r, i+\frac{1}{2}} \Delta v_{r, i+\frac{1}{2}} .
\end{aligned}
$$

This transformation states that the differential of $v^{2}\left(d v^{2}=2 v d v\right)$ is numerically exact in a second order accurate discretization. This is in fact the key to the success in the numerical integration by parts, since

$$
\begin{aligned}
& \sum_{i, j} \Delta \Omega_{i, j} \frac{v_{i, j}^{2}}{2}\left[\frac{\partial}{\partial \mathbf{v}} \cdot \mathbf{J}_{F P}\right]_{i, j} \\
& \quad=-\sum_{i, j}\left[\Delta v_{p, j+\frac{1}{2}} \Delta S_{i, j+\frac{1}{2}}\left(v_{p, j+\frac{1}{2}} R_{i, j+\frac{1}{2}}\right)+\Delta v_{r, i+\frac{1}{2}} \Delta S_{i+\frac{1}{2}, j}\left(v_{r, i+\frac{1}{2}} Z_{i+\frac{1}{2}, j}\right)\right] \\
& \quad=-\sum_{i, j}\left[\Delta \Omega_{i, j+\frac{1}{2}}\left(v_{p, j+\frac{1}{2}} R_{i, j+\frac{1}{2}}\right)+\Delta \Omega_{i+\frac{1}{2}, j}\left(v_{r, i+\frac{1}{2}} Z_{i+\frac{1}{2}, j}\right)\right]
\end{aligned}
$$

which is a numerical approximation of $-\int d \mathbf{v} \mathbf{v} \cdot \mathbf{J}_{F P}$, as desired. In Eq. (38), the volume elements $\Delta \Omega$ are given by

$$
\begin{aligned}
& \Delta \Omega_{i, j+\frac{1}{2}}=2 \pi v_{p, j+\frac{1}{2}} \Delta v_{p, j+\frac{1}{2}} \Delta v_{r, i} \\
& \Delta \Omega_{i+\frac{1}{2}, j}=2 \pi v_{p, j} \Delta v_{p, j} \Delta v_{r, i+\frac{1}{2}} .
\end{aligned}
$$


According to Eq. (30), the flux components $R_{i, j \pm 1 / 2}$ and $Z_{i \pm 1 / 2, j}$ will have two different contributions, namely, the contribution from the friction term (given by $(\partial / \partial \mathbf{v}) \cdot \bar{T}[H, H]$, that will henceforth be called the "T-term") and the contribution from the diffusion term (given by $(\partial / \partial \mathbf{v}) \cdot\left[\left(\partial^{2} G(f) / \partial \mathbf{v} \partial \mathbf{v}\right) f\right]$, and henceforth called the "G-term"). Thus,

$$
\begin{aligned}
& R=R_{g}+R_{t} \\
& Z=Z_{g}+Z_{t} .
\end{aligned}
$$

Each contribution is studied separately.

3.4.2. Discretization of the diffusion term of the Fokker-Planck flux ("G-term"). In cylindrical coordinates, the G-term reads

$$
\begin{gathered}
\frac{\partial}{\partial \mathbf{v}} \cdot\left[\frac{\partial^{2} G(f)}{\partial \mathbf{v} \partial \mathbf{v}} f\right]=R_{g} \mathbf{u}_{p}+Z_{g} \mathbf{u}_{r} \\
R_{g}=\frac{1}{v_{p}} \frac{\partial}{\partial v_{p}}\left(v_{p} \frac{\partial^{2} G}{\partial v_{p}^{2}} f\right)+\frac{\partial}{\partial v_{r}}\left(\frac{\partial^{2} G}{\partial v_{r} \partial v_{p}} f\right)-\frac{1}{v_{p}}\left(\frac{1}{v_{p}} \frac{\partial G}{\partial v_{p}} f\right) \\
Z_{g}=\frac{1}{v_{p}} \frac{\partial}{\partial v_{p}}\left(v_{p} \frac{\partial^{2} G}{\partial v_{r} \partial v_{p}} f\right)+\frac{\partial}{\partial v_{r}}\left(\frac{\partial^{2} G}{\partial v_{r}^{2}} f\right) .
\end{gathered}
$$

The $R_{g}$ and $Z_{g}$ components must be discretized so that the following integral (Eq. (11)) is reproduced numerically:

$$
\frac{1}{2} \int d \mathbf{v} \mathbf{v} \cdot \frac{\partial}{\partial \mathbf{v}} \cdot\left[\frac{\partial^{2} G(f)}{\partial \mathbf{v} \partial \mathbf{v}} f\right]=-\frac{1}{2} \int d \mathbf{v} f H .
$$

The integral in the left hand side is discretized as indicated in Eq. (38), but substituting $R_{i, j \pm 1 / 2}$ and $Z_{i \pm 1 / 2, j}$ by $R_{g i, j \pm 1 / 2}$ and $Z_{g i \pm 1 / 2, j}$, respectively. The components $R_{g}$ and $Z_{g}$ are discretized as

$$
\begin{aligned}
R_{g i, j \pm \frac{1}{2}}= & \underbrace{\frac{\left(G_{r p} f\right)_{i+\frac{1}{2}, j+\frac{1}{2}}-\left(G_{r p} f\right)_{i-\frac{1}{2}, j+\frac{1}{2}}}{\Delta v_{r, i}}+\underbrace{\frac{\left(v_{p} G_{p p} f\right)_{i, j+1}-\left(v_{p} G_{p p} f\right)_{i, j}}{v_{p, j+\frac{1}{2}} \Delta v_{p, j+\frac{1}{2}}}}_{R 2}}_{R 1} \\
& -\underbrace{\frac{\left(G_{p} f \Delta v_{p} / v_{p}^{*}\right)_{i, j+1}+\left(G_{p} f \Delta v_{p} / v_{p}^{*}\right)_{i, j}}{2 v_{p, j+\frac{1}{2}} \Delta v_{p, j+\frac{1}{2}}}}_{R 3} \\
Z_{g i \pm \frac{1}{2}, j}= & \underbrace{\frac{\left(v_{p} G_{r p} f\right)_{i+\frac{1}{2}, j+\frac{1}{2}}-\left(v_{p} G_{r p} f\right)_{i+\frac{1}{2}, j-\frac{1}{2}}}{v_{p, j} \Delta v_{p, j}}}_{Z 1}+\underbrace{\frac{\left(G_{r r} f\right)_{i+1, j}-\left(G_{r r} f\right)_{i, j}}{\Delta v_{r, i+\frac{1}{2}}}}_{Z 2} .
\end{aligned}
$$

Of particular interest is the discretization chosen for $\left(1 / v_{p}\right)\left(\left(1 / v_{p}\right)\left(\partial G / \partial v_{p}\right) f\right)$ in $R_{g}$ (term $R 3$ in $R_{g i, j+1 / 2}$ ). The term inside the parentheses has been averaged between the neighboring integer nodes $(i, j)$ and $(i, j+1)$, each weighed by the perpendicular velocity mesh spacing, and $v_{p, j}^{*}$ has been used instead of $v_{p, j}$. This form allows the sum to telescope to the desired result for an arbitrary mesh, at the cost of losing the second order accuracy for non-uniform meshes. 
The value of $f_{i \pm 1 / 2, j \pm 1 / 2}$, which appears in the $R 1$ and $Z 1$ terms, is calculated via a volume-weighed average of the values at the integer nodes:

$$
f_{i \pm \frac{1}{2}, j \pm \frac{1}{2}}=\frac{\Delta \Omega_{i, j} f_{i, j}+\Delta \Omega_{i \pm 1, j} f_{i \pm 1, j}+\Delta \Omega_{i, j \pm 1} f_{i, j \pm 1}+\Delta \Omega_{i \pm 1, j \pm 1} f_{i \pm 1, j \pm 1}}{\Delta \Omega_{i, j}+\Delta \Omega_{i \pm 1, j}+\Delta \Omega_{i, j \pm 1}+\Delta \Omega_{i \pm 1, j \pm 1}} .
$$

Better interpolation schemes have been devised [1] for the Fokker-Planck equation in spherical geometry (namely, exponential interpolation to incorporate the exponential nature of the distribution function). Unfortunately, the structure of the Fokker-Planck collision operator in cylindrical coordinates does not allow a straightforward implementation of such interpolation schemes, and this issue remains to be an open one. At any rate, whatever the interpolation scheme selected for $f_{i \pm 1 / 2, j \pm 1 / 2}$, it is of paramount importance that the interpolation coefficients of the values of the distribution function at the four corners be symmetric, to allow the adequate cancellation of the discretized integrals (see sums of $R 1$ and $Z 1$ terms below).

Note that the term $Z 1$ is singular when $v_{p} \rightarrow 0(j=1)$. This singularity is resolved by L'hospital's rule (in the same way as done in Eqs. (58) and (59) in Appendix A), yielding

$$
\left.\frac{\left(v_{p} G_{r p} f\right)_{i+\frac{1}{2}, j+\frac{1}{2}}-\left(v_{p} G_{r p} f\right)_{i+\frac{1}{2}, j-\frac{1}{2}}}{v_{p, j} \Delta v_{p, j}}\right|_{j=1} \rightarrow \frac{4\left(G_{r p} f\right)_{i+\frac{1}{2}, \frac{3}{2}}}{\Delta v_{p, 1}} .
$$

Symmetry in the term $R 1$ at $i=1$ is imposed as follows (refer to Appendix A for details):

$$
\left.\frac{\left(G_{r p} f\right)_{i+\frac{1}{2}, j+\frac{1}{2}}-\left(G_{r p} f\right)_{i-\frac{1}{2}, j+\frac{1}{2}}}{\Delta v_{r, i}}\right|_{i=1} \rightarrow \frac{2\left(G_{r p} f\right)_{\frac{3}{2}, j+\frac{1}{2}}}{\Delta v_{r, 1}} .
$$

The sum in Eq. (38), specialized for the components of the G-term, is performed separately for each term in $R_{g i, j+\frac{1}{2}}$ and $Z_{g i+\frac{1}{2}, j}$ :

- Term R1,

$$
\begin{aligned}
& \sum_{i, j} \Delta v_{p, j+\frac{1}{2}} v_{p, j+\frac{1}{2}}^{2}\left[\left(G_{r p} f\right)_{i+\frac{1}{2}, j+\frac{1}{2}}-\left(G_{r p} f\right)_{i-\frac{1}{2}, j+\frac{1}{2}}\right] \\
& =\sum_{j} \Delta v_{p, j+\frac{1}{2}} v_{p, j+\frac{1}{2}}^{2} \underbrace{\sum_{i}\left[\left(G_{r p} f\right)_{i+\frac{1}{2}, j+\frac{1}{2}}-\left(G_{r p} f\right)_{i-\frac{1}{2}, j+\frac{1}{2}}\right]}_{=0}=0 .
\end{aligned}
$$

- Term R2,

$$
\begin{aligned}
& \sum_{i, j} \Delta v_{r, i} v_{p, j+\frac{1}{2}}\left[v_{p, j+1} G_{p p i, j+1} f_{i, j+1}-v_{p, j} G_{p p i, j} f_{i, j}\right] \\
& =\sum_{i, j} \Delta v_{r, i} v_{p, j} G_{p p i, j} f_{i, j} \overbrace{\left[v_{p, j-\frac{1}{2}}-v_{p, j+\frac{1}{2}}\right]}^{-\Delta v_{p, j}} \\
& =-\sum_{i, j} \Delta v_{r, i} \Delta v_{p, j} v_{p, j} G_{p p i, j} f_{i, j} .
\end{aligned}
$$


- Term R3,

$$
\begin{aligned}
& -\sum_{i, j} \Delta v_{r, i} v_{p, j+\frac{1}{2}} \frac{1}{2}\left(\frac{G_{p i, j+1} f_{i, j+1} \Delta v_{p, j+1}}{v_{p, j+1}^{*}}+\frac{G_{p i, j} f_{i, j} \Delta v_{p, j}}{v_{p, j}^{*}}\right) \\
& =-\sum_{i, j} \Delta v_{r, i} \frac{G_{p i, j} f_{i, j} \Delta v_{p, j}}{v_{p, j}^{*}} \underbrace{\left(\frac{v_{p, j-\frac{1}{2}}+v_{p, j+\frac{1}{2}}}{2}\right)}_{v_{p, j}^{*}} \\
& =-\sum_{i, j} \Delta v_{r, i} \Delta v_{p, j} G_{p i, j} f_{i, j} .
\end{aligned}
$$

- $\operatorname{Term} \mathrm{Z1}$,

$$
\begin{aligned}
& \sum_{i, j} \Delta v_{r, i+\frac{1}{2}} v_{r, i+\frac{1}{2}}\left[\left(v_{p} G_{r p} f\right)_{i+\frac{1}{2}, j+\frac{1}{2}}-\left(v_{p} G_{r p} f\right)_{i+\frac{1}{2}, j-\frac{1}{2}}\right] \\
& =\sum_{i} \Delta v_{r, i+\frac{1}{2}} v_{r, i+\frac{1}{2}} \underbrace{\sum_{j}\left[\left(v_{p} G_{r p} f\right)_{i+\frac{1}{2}, j+\frac{1}{2}}-\left(v_{p} G_{r p} f\right)_{i+\frac{1}{2}, j-\frac{1}{2}}\right]}_{=0}=0 .
\end{aligned}
$$

- Term Z2,

$$
\begin{aligned}
& \sum_{i, j} v_{p, j} \Delta v_{p, j} v_{r, i+1 / 2}\left(G_{r r i+1, j} f_{i+1, j}-G_{r r i, j} f_{i, j}\right) \\
& =\sum_{i, j} \Delta v_{r, i} v_{p, j} G_{r r i, j} f_{i, j} \overbrace{\left[v_{r, i-\frac{1}{2}}-v_{r, i+\frac{1}{2}}\right]}^{-\Delta v_{r, i}} \\
& =-\sum_{i, j} \Delta v_{r, i} \Delta v_{p, j} v_{p, j} G_{r r i, j} f_{i, j} .
\end{aligned}
$$

These sums are exact because the boundary terms are zero at both the inner boundaries (by symmetry) and the outer boundaries (because $f=0$ if the distribution function is absolutely confined in the domain). Then, the discretization of the G-term satisfies

$$
\begin{aligned}
\frac{1}{2} \int d \mathbf{v} \mathbf{v} \cdot \frac{\partial}{\partial \mathbf{v}} \cdot\left[\frac{\partial^{2} G(f)}{\partial \mathbf{v} \partial \mathbf{v}} f\right] & \approx \sum_{i, j}\left[\Delta \Omega_{i, j+\frac{1}{2}}\left(v_{p, j+\frac{1}{2}} R_{g i, j+\frac{1}{2}}\right)+\Delta \Omega_{i+\frac{1}{2}, j}\left(v_{r, i+\frac{1}{2}} Z_{g i+\frac{1}{2}, j}\right)\right] \\
& =-\frac{1}{2} \sum_{i, j} \Delta v_{r, i} \Delta v_{p, j} v_{p, j} \underbrace{\left[G_{p p}+\frac{G_{p}}{v_{p}}+G_{r r}\right]}_{\nabla_{\mathbf{v}}^{2} G=H} f_{i, j} \\
& =-\sum_{i, j} \Delta v_{r, i} \Delta v_{p, j} v_{p, j} \frac{H_{i, j} f_{i, j}}{2}
\end{aligned}
$$

which is the numerical approximation of $-\frac{1}{2} \int d \mathbf{v} f H=\int d \mathbf{v} Q[H, H]$, as desired. Note that the previous result requires that

$$
\left[G_{p p}+\frac{G_{p}}{v_{p}}+G_{r r}\right]_{i, j}=H_{i, j}
$$


be satisfied numerically. Thus, the conservative discretization of the G-term has rendered the adequate numerical formulation of the $G$ problem (which, in this case, is the obvious one; this, however, will not be the case for the $H$ Rosenbluth potential, as will be clear shortly).

3.4.3. Discretization of the friction term of the Fokker-Planck flux ("T-term"). Define $(\partial / \partial \mathbf{v}) \cdot \bar{T}[H, H]=R_{t} \mathbf{u}_{p}+Z_{t} \mathbf{u}_{r}$, where $R_{t}$ and $Z_{t}$ are expressed in cylindrical coordinates as

$$
\begin{aligned}
& R_{t}=\frac{1}{8 \pi}\left[\frac{1}{2} \frac{\partial H_{p}^{2}}{\partial v_{p}}+\frac{H_{p}^{2}}{v_{p}}-\frac{1}{2} \frac{\partial H_{r}^{2}}{\partial v_{p}}+\frac{\partial}{\partial v_{r}}\left(H_{r} H_{p}\right)\right] \\
& Z_{t}=\frac{1}{8 \pi}\left[-\frac{1}{2} \frac{\partial H_{p}^{2}}{\partial v_{r}}+\frac{1}{2} \frac{\partial H_{r}^{2}}{\partial v_{r}}+\frac{1}{v_{p}} \frac{\partial}{\partial v_{r}}\left(v_{p} H_{r} H_{p}\right)\right] .
\end{aligned}
$$

These components are discretized in the following way:

$$
\begin{aligned}
R_{t i, j+\frac{1}{2}}= & \frac{1}{8 \pi}\left[\frac{1}{2} \frac{H_{p i, j+1}^{2}-H_{p i, j}^{2}}{\Delta v_{p, j+\frac{1}{2}}}+\frac{H_{p i, j+\frac{1}{2}}^{2}}{v_{p, j+\frac{1}{2}}}-\frac{1}{2} \frac{H_{r i, j+1}^{2}-H_{r i, j}^{2}}{\Delta v_{p, j+\frac{1}{2}}}\right. \\
& \left.+\frac{\left.\left(H_{r} H_{p}\right)_{i+\frac{1}{2}, j+\frac{1}{2}}-\left(H_{r} H_{p}\right)_{i-\frac{1}{2}, j+\frac{1}{2}}\right]}{\Delta v_{r, i}}\right] \\
Z_{t i+\frac{1}{2}, j}= & \frac{1}{8 \pi}\left[\frac{v_{p, j}^{*}}{v_{p, j}}\left(-\frac{1}{2} \frac{H_{p i+1, j}^{2}-H_{p i, j}^{2}}{\Delta v_{r, i+\frac{1}{2}}}+\frac{1}{2} \frac{H_{r i+1, j}^{2}-H_{r i, j}^{2}}{\Delta v_{r, i+\frac{1}{2}}}\right)\right. \\
& \left.+\frac{\left(v_{p} H_{r} H_{p}\right)_{i+\frac{1}{2}, j+\frac{1}{2}}-\left(v_{p} H_{r} H_{p}\right)_{i+\frac{1}{2}, j-\frac{1}{2}}}{v_{p, j} \Delta v_{p, j}}\right] .
\end{aligned}
$$

Note that a non-physical correction factor, $v_{p, j}^{*} / v_{p, j}$, has been introduced in $Z_{t i+1 / 2, j}$ to allow the sum to telescope to the desired result for an arbitrary mesh. It is relevant only for non-uniform meshes. Introducing $R_{t i, j+1 / 2}$ and $Z_{t i+1 / 2, j}$ into Eq. (38) (instead of $R_{i, j \pm 1 / 2}$ and $Z_{i \pm 1 / 2, j}$ ), we obtain

$$
\begin{aligned}
\int d \mathbf{v} \mathbf{v} \cdot \frac{\partial}{\partial \mathbf{v}} \cdot \bar{T}[H, H] \approx & \sum_{i, j}\left[\Delta \Omega_{i, j+\frac{1}{2}}\left(v_{p, j+\frac{1}{2}} R_{t i, j+\frac{1}{2}}\right)+\Delta \Omega_{i+\frac{1}{2}, j}\left(v_{r, i+\frac{1}{2}} Z_{t i+\frac{1}{2}, j}\right)\right] \\
= & \frac{1}{8 \pi} \sum_{i, j}\left[-\frac{1}{2} \Delta v_{r, i} v_{p, j} \Delta v_{p, j} H_{p i, j}^{2}+\Delta v_{r, i} v_{p, j+\frac{1}{2}} \Delta v_{p, j+\frac{1}{2}} H_{p, i, j+\frac{1}{2}}^{2}\right. \\
& \left.+\frac{1}{2} \Delta v_{r, i} v_{p, j} \Delta v_{p, j} H_{r i, j}^{2}\right]+ \text { B.T.s }
\end{aligned}
$$

which is an approximate from of the theoretical result in Eq. (16). Here, B.T.s represents the boundary terms left unpaired after telescoping the sum.

3.4.4. Discretization of $Q[H, H]$ for the cancellation of the energy moment within the domain. The necessary and sufficient condition for numerical energy conservation is

$\sum_{i, j} \Delta \Omega_{i, j} \frac{v_{i, j}^{2}}{2}\left[\frac{\partial}{\partial \mathbf{v}} \cdot \mathbf{J}_{F P}\right]_{i, j}=-\sum_{i, j}\left[\Delta \Omega_{i, j+\frac{1}{2}}\left(v_{p, j+\frac{1}{2}} R_{i, j+\frac{1}{2}}\right)+\Delta \Omega_{i+\frac{1}{2}, j}\left(v_{r, i+\frac{1}{2}} Z_{i+\frac{1}{2}, j}\right)\right]=0$ 
which, after introducing Eqs. (43) and (44), reads

$$
\begin{aligned}
& \frac{1}{8 \pi} \sum_{i, j}\left[-\frac{1}{2} \Delta v_{r, i} v_{p, j} \Delta v_{p, j} H_{p, i, j}^{2}+\Delta v_{r, i} v_{p, j+\frac{1}{2}} \Delta v_{p, j+\frac{1}{2}} H_{p, i, j+\frac{1}{2}}^{2}\right. \\
& \left.+\frac{1}{2} \Delta v_{r, i} v_{p, j} \Delta v_{p, j} H_{r i, j}^{2}\right]=\sum_{i, j} \Delta v_{r, i} \Delta v_{p, j} v_{p, j} \frac{H_{i, j} f_{i, j}}{2}
\end{aligned}
$$

plus boundary terms, which are neglected at this stage. Recalling the definition of $Q[H, H]$ (Eq. (12)), we can write

$$
\frac{H f}{2}=-\frac{H \nabla_{\mathbf{v}}^{2} H}{16 \pi}=-Q[H, H]
$$

Hence, exact numerical energy conservation requires

$$
\begin{aligned}
& \frac{1}{8 \pi} \sum_{i, j}\left[-\frac{1}{2} \Delta v_{r, i} v_{p, j} \Delta v_{p, j} H_{p i, j}^{2}+\Delta v_{r, i} v_{p, j+\frac{1}{2}} \Delta v_{p, j+\frac{1}{2}} H_{p i, j+\frac{1}{2}}^{2}\right. \\
& \left.\quad+\frac{1}{2} \Delta v_{r, i} v_{p, j} \Delta v_{p, j} H_{r i, j}^{2}\right]=-\sum_{i, j} \Delta v_{r, i} \Delta v_{p, j} v_{p, j} Q_{i, j}[H, H] .
\end{aligned}
$$

This identity is possible if $Q[H, H]$ is rearranged as

$Q[H, H]=\frac{H \nabla_{\mathbf{v}}^{2} H}{16 \pi}=\frac{1}{16 \pi}\left[\frac{\partial}{\partial v_{r}}\left(H_{r} H\right)-H_{r}^{2}+2 \frac{H}{v_{p}} \frac{\partial}{\partial v_{p}}\left(v_{p} H_{p}\right)+H_{p}^{2}-\frac{1}{v_{p}} \frac{\partial}{\partial v_{p}}\left(v_{p} H_{p} H\right)\right]$ which, once discretized, reads as

$$
\begin{aligned}
Q_{i, j}[H, H]= & \frac{1}{16 \pi}\left\{\frac{\left(H_{r} H\right)_{i+\frac{1}{2}, j}-\left(H_{r} H\right)_{i-\frac{1}{2}, j}}{\Delta v_{r, i}}-\frac{v_{p, j}^{*}}{v_{p, j}} H_{r i, j}^{2}\right. \\
& +\frac{2 H_{i, j}}{v_{p, j}} \frac{\left(v_{p} H_{p}\right)_{i, j+\frac{1}{2}}-\left(v_{p} H_{p}\right)_{i, j-\frac{1}{2}}}{\Delta v_{p, j}}+\frac{v_{p, j}^{*}}{v_{p, j}} H_{p i, j}^{2} \\
& \left.-\frac{\left(v_{p} H_{p} H\right)_{i, j+\frac{1}{2}}-\left(v_{p} H_{p} H\right)_{i, j-\frac{1}{2}}}{v_{p, j} \Delta v_{p, j}}\right\} .
\end{aligned}
$$

This result renders Eq. (45) exact within the velocity domain, but fails at the boundaries due to "unpaired" boundary terms. Theoretically, these boundary terms exactly cancel each other (Eqs. (19), (20)); however, numerically, they do not. This result reflects an intrinsic limitation of all numerical techniques in Fokker-Planck problems, namely, the impossibility of considering infinite domains. Consequently, exact energy conservation in Fokker-Planck problems is not possible, although the error can be kept small by taking advantage of the scaling laws of the mismatch at the boundary. This issue is discussed further in the next section.

Note that the correction factor $v_{p, j}^{*} / v_{p, j}$ has been introduced again to allow the sum to telescope to the desired result for an arbitrary mesh. In Eq. (48), $H_{r}$ and $H_{p}$ are discretized using centered finite differences on the integer mesh, and $H_{i, j+1 / 2}$ and $H_{i+1 / 2, j}$ are calculated as the average of the values at the neighboring integer mesh points. 
In order for Eq. (45) to cancel, $H$ must be obtained from Eq. (46) (discretized as indicated in Eq. (48)), a non-linear equation that has to be solved iteratively using Newton's method, instead of using its (simpler) linear definition, $\nabla_{\mathbf{v}}^{2} H=-8 \pi f$. Both problems (linear and non-linear) have in fact identical solutions in the continuum, but their numerical solutions differ by truncation errors. The advantage of the non-linear representation is that, as shown above, it leads to cancellation of the energy moment integral (thus imporving energy conservation).

This subtle digression from the traditional treatment of $H$ is in fact crucial in the development of an energy-conservative method, as will be shown in the numerical evidence presented in Section 5. The added computational expense that the non-linear problem reprsents is minimized by using the numerical solution of the linear $H$-problem as the initial guess for Newton's algorithm. Thus, the non-linear correction is of the order of the truncation error of the discretization, and Newton's algorithm typically converges in less than five iterations in spite of very stringent convergence tolerances $\left(\left\|\mathbf{e}_{r}\right\|_{2}<10^{-9}\right.$, where $\mathbf{e}_{r}$ is the vector of residuals).

\section{SCALING OF THE ENERGY ERROR}

This section presents a heuristic theoretical model to explain the scaling of the error in the cancellation of the energy moment with $v_{\text {limit }}$ and $N$, as observed in the results presented in Section 5.

From the previous discussion, it is clear that the cancellation of the energy moment is precluded by errors in the cancellation of boundary terms. These boundary terms are represented theoretically by the boundary integrals in Eq. (18), reproduced as

$$
\frac{\partial E}{\partial t} \propto \underbrace{\oint_{\partial \Omega} d \mathbf{S} \cdot \frac{1}{2} \mathbf{v}\left(\frac{\partial H}{\partial \mathbf{v}}\right)^{2}}_{I_{1}}-\underbrace{\oint_{\partial \Omega} d \mathbf{S} \cdot \frac{\partial H}{\partial \mathbf{v}}\left(\mathbf{v} \cdot \frac{\partial H}{\partial \mathbf{v}}+\frac{H}{2}\right)}_{I_{2}}=0 .
$$

The mismatch is caused by second order truncation errors in the determination of the gradient of $H$ and the integration procedure itself, each contribution of comparable magnitude. However, for the sake of heuristically understanding how this error behaves, only the effect of the truncation term of the gradient will be studied. In a centered finite difference scheme, the gradient of the $H$ Rosenbluth potential is approximated by the following truncated Taylor expansion in velocity space $\left(v_{r}, v_{p}\right)$,

$$
\left.\frac{\partial H}{\partial \mathbf{v}} \simeq \frac{\partial H}{\partial \mathbf{v}}\right|_{0}+\left.\frac{1}{2}\left[\Delta v_{r} \frac{\partial}{\partial v_{r}}+\Delta v_{p} \frac{\partial}{\partial v_{p}}\right]^{(2)} \frac{\partial H}{\partial \mathbf{v}}\right|_{0}
$$

The superscript (2) means exponent for the increments, and order for the derivatives; the subscript 0 means exact value. Note that the angular coordinate does not appear because the problem is symmetric with respect to the rotation axis (given by $\mathbf{r}$ in Fig. 1).

If the numerical errors in the approximations of $I_{1}$ and $I_{2}$ in Eq. (49) are represented by $\Delta_{1}$ and $\Delta_{2}$, respectively, the resultant error in the energy moment integral (represented by $\Delta_{E}$ ) can be expressed as

$$
\Delta_{E}=\Delta_{1}-\Delta_{2} .
$$


(Eqs. (19) and (20)), and hence expected to behave similarly. The leading term of the error introduced in $I_{1}$ due to a second order approximation of the gradient reads

$$
\left.\Delta_{1} \sim K \int_{\partial \Omega} d \mathbf{S} \cdot \mathbf{v}\left(\left.\frac{1}{2}\left[\Delta v_{r} \frac{\partial}{\partial v_{r}}+\Delta v_{p} \frac{\partial}{\partial v_{p}}\right]^{(2)} \frac{\partial H}{\partial \mathbf{v}}\right|_{0}\right) \cdot \frac{\partial H}{\partial \mathbf{v}}\right|_{0}+O\left(\Delta v^{4}\right),
$$

where $K$ is some proportionality constant. If $v_{\text {limit }} \gg v_{0}$, where $v_{0}$ is the characteristic speed in the system (usually the thermal velocity in equilibrium), then the distribution function can be considered a point source, and $H_{0} \sim \frac{1}{v}$, where $v=\sqrt{v_{r}^{2}+v_{p}^{2}}$. Then, the derivatives in Eq. (52) can be calculated analytically, and the integral at the boundaries can be performed. This has been done in Appendix B for three different cases: uniform mesh, geometric mesh, and a combined uniform/geometric mesh.

Note that the accuracy of the numerical representation of the distribution function in the limit of $v_{\text {limit }} \gg v_{0}$ might be compromised, because $f \sim e^{-\left(v / v_{o}\right)^{2}}$, and actual values of $f$ may fall out of the machine precision range. However, because the absolute values taken by $f$ in this region are extremely small, the effect of large round-off errors when $v_{\text {limit }} \gg v_{0}$ on the accuracy of the global solution is expected to be minimal (i.e., having $f \sim 10^{-16}$ instead of $f \sim 10^{-25}$ will have very little impact on the solution in the region of interest, where $f \sim 1$ ). In addition, note from Eq. (47) that the numerical cancellation of the energy moment relies on $H$, which scales as $\frac{1}{v}$ and hence can be calculated much more accurately.

\subsection{Scaling Laws for $\Delta_{E}$ in a Uniform Mesh}

The velocity increments in a uniform mesh are defined by $\Delta v_{r}=v_{\text {limit }} / N_{r}$ and $\Delta v_{p}=$ $v_{\text {limit }} / N_{p}$, where $N_{r} * N_{p}=N$, the total number of mesh points. The integral in Eq. (52) yields, for this case (Appendix B),

$$
\Delta_{1} \sim-\frac{v_{0}\left(\Delta v_{r}^{2}+1.4 \Delta v_{p}^{2}+3.8 \Delta v_{r} \Delta v_{p}\right)}{v_{\text {limit }}^{3}} \sim \frac{v_{0}}{N v_{\text {limit }}},
$$

where it has been assumed that $N_{r} \sim N_{p} \sim \sqrt{N}$. Hence, as $\Delta_{2}$ behaves similarly, we expect

$$
\Delta_{E}=\Delta_{1}-\Delta_{2} \sim \frac{v_{0}}{N v_{\text {limit }}} .
$$

This result indicates that, for a uniform mesh, the error in the energy rate of change decreases as the number of mesh points increases, and decreases as the outer boundaries are pushed further. Note that these are not competing trends, because the result in Eq. (53) does not depend on the increments themselves. In particular, the energy error will decrease even when $v_{\text {limit }}$ is increased and $N$ is left constant, even though the mesh gets coarser. Of course, this is true as long as $\Delta v_{r}$ and $\Delta v_{p}$ remain sufficiently small to prevent higher order terms $\left[O\left(\Delta v^{4}\right)\right]$ becoming significant.

\subsection{Scaling Laws for $\Delta_{E}$ in a Geometric Mesh}

A geometric velocity mesh, characterized by a geometric ratio $\xi$, is defined by

$$
\begin{aligned}
\Delta v_{k} & =\xi \Delta v_{k-1}=\xi^{k-1} \Delta v_{1} \\
v_{k} & =\sum_{n=1}^{k} \Delta v_{n}=\frac{\xi^{k-1}}{\xi-1} \Delta v_{1}
\end{aligned}
$$


where $k=2, \ldots, N_{r}$ for $v_{r}$, and $k=2, \ldots, N_{p}$ for $v_{p}$. The error in the boundary integral yields (Appendix B)

$$
\Delta_{1} \sim-C(\xi) \frac{v_{0}(\xi-1)^{2}}{v_{\text {limit }}},
$$

where $C(\xi)$ is a slowly varying function of $\xi: C(\xi \rightarrow 1) \simeq 5, C(\xi=2) \simeq 2.23$. Since $\Delta_{2}$ is expected to behave identically, the error in the energy rate of change scales as

$$
\Delta_{E} \sim \frac{v_{0}(\xi-1)^{2}}{v_{\text {limit }}}=\frac{\epsilon^{2} v_{0}}{v_{\text {limit }}},
$$

where $\epsilon=\xi-1$. Note that this expression does not hold for $\epsilon=0$ (uniform mesh limit), due to the approximations made in the integration procedure in Appendix B (where it is assumed that $\xi^{\sqrt{N}} \gg 1$, or, equivalently, $\epsilon \geq 1 / \sqrt{N}$ ).

Note that most geometric ratios in a reasonably fine mesh will not go beyond $\xi \sim 1.1$. Hence, $\xi^{x}=(1+\epsilon)^{x} \approx 1+x \epsilon+\frac{x(x-1)}{2} \epsilon^{2}$, and, according to the definition of the geometric mesh, we can write

$$
v_{\text {limit }}=\frac{\xi^{\sqrt{N}}-1}{\xi-1} \Delta v_{1} \approx\left(1+\frac{\sqrt{N} \epsilon}{2}\right) \sqrt{N} \Delta v_{1}
$$

where $\Delta v_{1}$ is the first velocity increment in the geometric mesh, chosen so that the mesh provides enough resolution in the region where the bulk of the distribution function is located. In actual simulations, this usually results in $\sqrt{N} \Delta v_{1} \sim 5 v_{0}$ (where $v_{0}$ is the characteristic speed in the system), and hence

$$
\epsilon \sim \frac{2}{\sqrt{N}}\left[\frac{v_{\text {limit }}}{5 v_{0}}-1\right]
$$

This result is compatible with $\epsilon \ll 1$ only if $v_{\text {limit }} / 5 v_{0} \ll \sqrt{N}$, which is typically the case (for instance, in a $32 \times 32$ mesh, $v_{\text {limit }}$ would have to be $>160 v_{0}$ to violate this condition). Hence, in regards to $\epsilon$, two situations are possible:

- $\epsilon \sim 1 / \sqrt{N} \ll 1$. This is the case when $v_{\text {limit }}$ is only a few times larger than $5 v_{0}$. The error in the energy rate of change scales as

$$
\Delta_{E} \sim \frac{v_{0}}{N v_{\text {limit }}},
$$

i.e., it decreases with both $v_{\text {limit }}$ and $N$. Note that this scaling is consistent with the uniform mesh scaling; hence, it can be expected to apply also for $0<\epsilon<1 / \sqrt{N}$.

- $\epsilon \sim v_{\text {limit }} / \sqrt{N} v_{0} \ll 1$. This is the case when $v_{\text {limit }} \gg 5 v_{0}$, and the energy error scales as

$$
\Delta_{E} \sim \frac{v_{\text {limit }}}{N v_{0}}
$$

The favorable scaling with the velocity domain limit is lost, as the energy error increases with $v_{\text {limit }}$. Larger values of $\epsilon$ are not of interest, because accuracy would be lost, and higher order terms in Eq. (52) would become significant, thus breaking the scaling. 


\subsection{Scaling of $\Delta_{E}$ in Combined Uniform-Geometric Meshes}

The optimal choice will come from combining the two previous cases so that:

1. Enough resolution, with a well-known accuracy in the numerical method, is provided in the domain region where the distribution function is localized.

2. Large $v_{\text {limits }}$ are possible without requiring a large number of mesh points $N$.

3. The energy error decreases with both increasing domain limits and increasing accuracy.

4. The influence in the numerical solution of the non-physical correction factor $v_{p, j}^{*} /$ $v_{p, j}$, introduced in the discretization to allow the exact numerical cancellation of the energy moment, is minimized.

Optimization will be achieved by constructing a mesh that discretizes the velocity domain in the following way:

- The important physics in the problem will occur in the domain region limited by $v_{r}, v_{p} \in\left(0,5 v_{0}\right)$, where $v_{0}$ is the characteristic velocity in the problem, typically defined as the thermal velocity in equilibrium. Hence, to provide accuracy and resolution, this region will be discretized with a uniform mesh.

- The rest of the domain up to the outer boundaries will be discretized with a geometric mesh with initial velocity increment equal to the increment of the uniform mesh, to ensure a smooth transition between both meshes.

- The increments of the last two nodes in both velocity axes are set equal to the uniform velocity increment, in an attempt to improve the scaling of the energy error by increasing the accuracy at the outer boundaries.

The number of mesh points in the uniform and the geometric portions of the domain is comparable (for instance, in a $\frac{1}{2} / \frac{1}{2}$ or $\frac{1}{3} / \frac{2}{3}$ proportion). A sketch of the discretization of the velocity domain that results is depicted in Fig. 4.

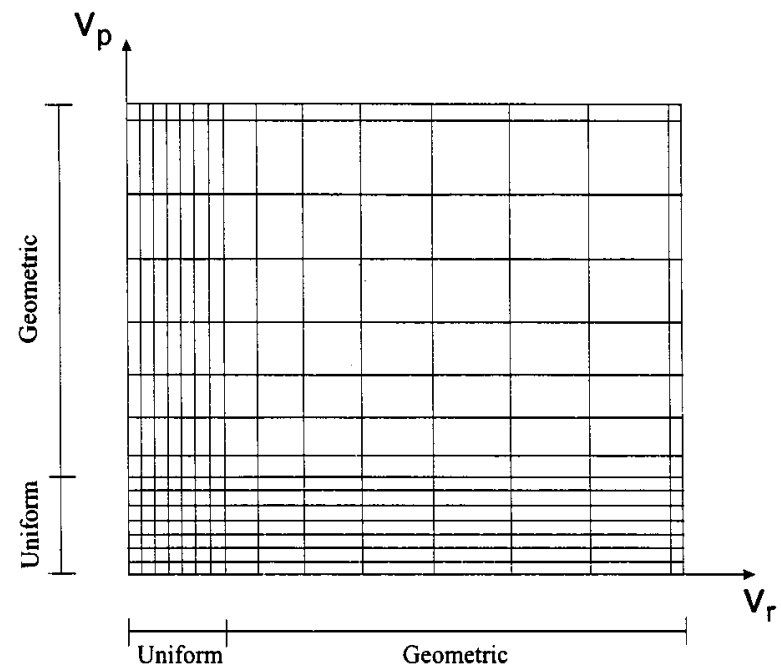

FIG. 4. Sketch of a combined uniform-geometric discretization mesh. Note the additional accuracy provided at the outer boundaries. 
The error of the boundary integral in this combined domain is also obtained in Appendix B, and yields

$$
\Delta_{1} \sim \frac{\epsilon^{2} v_{0}}{v_{\text {limit }}}\left[-\frac{1}{2}-\frac{5}{2}\left(\frac{5 v_{0}}{\epsilon \sqrt{N} v_{\text {limit }}}\right)-\frac{5}{3}\left(\frac{5 v_{0}}{\epsilon \sqrt{N} v_{\text {limit }}}\right)^{2}\right]
$$

This result applies for $\epsilon \geq 1 / \sqrt{N} \ll 1$. The result in Eq. (55) presents an expansion of the error in terms of the parameter $5 v_{0} / \epsilon \sqrt{N} v_{\text {limit }}$, which behaves as

$$
\begin{aligned}
& \epsilon \sim \frac{1}{\sqrt{N}}\left(v_{\text {limit }}>5 v_{0}\right) \Rightarrow \frac{5 v_{0}}{\epsilon \sqrt{N} v_{\text {limit }}} \sim \frac{5 v_{0}}{v_{\text {limit }}}<1 \\
& \epsilon \sim \frac{v_{\text {limit }}}{\sqrt{N} v_{0}}\left(v_{\text {limit }} \gg 5 v_{0}\right) \Rightarrow \frac{v_{0}}{\epsilon \sqrt{N} v_{\text {limit }}} \sim\left(\frac{5 v_{0}}{v_{\text {limit }}}\right)^{2} \ll 1,
\end{aligned}
$$

using the $\epsilon$ scaling results of the previous subsection. These relations are revealing for understanding the scaling of the error of the energy moment integral, $\Delta_{E}=\Delta_{1}-\Delta_{2}$ :

- $\epsilon \sim 1 / \sqrt{N} \ll 1$. In principle, the first term in the expansion dominates, and hence, the error in the energy rate of change scales as

$$
\Delta_{E} \sim \frac{\epsilon^{2} v_{0}}{v_{\text {limit }}} \sim \frac{v_{0}}{N v_{\text {limit }}}
$$

However, since $\Delta_{E}=\Delta_{1}-\Delta_{2}$, and $\Delta_{1}$ and $\Delta_{2}$ originate from different forms of the same integral, they are expected to behave similarly to first order, and the leading terms in the expansion may cancel to some degree. This, together with the fact that the coefficient of the leading term in the expansion is already small vs that of the first order term, suggests that the latter may actually dominate the scaling of $\Delta_{E}$ in some cases. Thus, in some cases it may occur that

$$
\Delta_{E} \sim \frac{\epsilon v_{0}^{2}}{\sqrt{N} v_{\text {limit }}^{2}} \sim \frac{1}{N}\left(\frac{v_{0}}{v_{\text {limit }}}\right)^{2}
$$

Some numerical evidence of this quadratic scaling is presented in Section 5. Hence,

$$
\Delta_{E} \sim\left[\frac{v_{0}}{N v_{\text {limit }}}, \frac{1}{N}\left(\frac{v_{0}}{v_{\text {limit }}}\right)^{2}\right]
$$

Note that, at any rate, the uniform mesh scaling has to be recovered when $\epsilon \rightarrow 0$ (uniform mesh limit).

- $\epsilon \sim v_{\text {limit }} / \sqrt{N} v_{0} \ll 1$. In this case, the energy error may scale as

$$
\Delta_{E} \sim\left[\frac{v_{\text {limit }}}{N v_{0}}, \frac{v_{0}}{N v_{\text {limit }}}\right]
$$




\section{TABLE I}

Scaling Laws of $\Delta_{E}$ in Terms of $v_{\text {limit }}$ and $N$ as a Function of $\epsilon=\xi-1$ (where $\xi$ Is the Geometric Ratio), for uniform, Geometric, and Combined Meshes

\begin{tabular}{lcc}
\hline Scaling of $\Delta_{E}$ & $\epsilon \sim \frac{1}{\sqrt{N}} \ll 1$ & $\epsilon \sim \frac{v_{\text {limit }}}{\sqrt{N v_{0}} \ll 1}$ \\
\hline Uniform mesh & $\frac{v_{0}}{N v_{\text {limit }}}$ & $\frac{v_{0}}{N v_{\text {limit }}}$ \\
Geometric mesh & $\frac{v_{0}}{N v_{0}}$ \\
Combined mesh & $\frac{v_{0}^{2}}{N v_{\text {limit }}^{2}}$ & $\frac{v_{\text {limit }}}{N v_{0}}, \frac{v_{0}}{N v_{\text {limit }}}$ \\
\hline
\end{tabular}

\subsection{Summary of the Scaling Laws of $\Delta_{E}$ for Different Meshes}

Table I summarizes the scaling laws of $\Delta_{E}$ obtained in the previous paragraphs, for the three different meshes considered, namely, uniform, geometric, and combined mesh. This table confirms that the combined mesh is the optimal choice, since it provides scalings as good as those of the uniform mesh (for $\epsilon \sim 1 / \sqrt{N} \ll 1$ ), and is much more flexible. However, it should be borne in mind that the truncation error of the discretization of the Fokker-Planck equation is only first order accurate in the geometric (nonuniform) subdomain (centered finite differences are second order accurate only in uniform meshes), and the accuracy of the numerical representation increases as $\epsilon$ decreases Hence, the final selection of the velocity domain and its discretization mesh must observe a certain compromise between energy conservation and the accuracy of the numerical method.

\section{RESULTS}

The goal of this section is twofold, namely, (1) compare the performance of the energyconservative discretization with that of standard second-order, particle-conservative discretization techniques, and (2) show the numerical scaling of $\Delta_{E}$ with $v_{\text {limit }}$ and $N$, and compare them with the theoretical trends derived in Section 4. For this purpose, a 2D cylindrical velocity domain is considered, discretized with a combined uniform-geometric mesh with $\sqrt{N}$ points per direction ( $N$ total) split in a $\frac{1}{2} / \frac{1}{2}$ proportion between the uniform and geometric regions. Two additional mesh points, spaced with the uniform grid spacing, provide extra accuracy at the outer boundaries. The uniform mesh region is limited by $v_{r}, v_{p} \in[0, \sqrt{2}]$; the geometric mesh region is limited by $v_{r}, v_{p} \in\left[\sqrt{2}, v_{\text {limit }}\right]$; velocities are in units of an arbitrary reference velocity, $v_{0}$.

Two distinct test distribution functions will be considered for the numerical experiments in order to illustrate a range of characteristic problems:

1. A radial beam, characterized by a distribution function $f\left(v_{r}, v_{p}\right)$ centered on $v_{r}=$ 0.5 and $v_{p}=0$, with beam temperature $T_{b}=8.89 \cdot 10^{-3}$ and average energy $\langle E\rangle=0.138$.

2. A symmetric beam, characterized by $f\left(v_{r}, v_{p}\right)=f(v)$, centered on $v=\sqrt{v_{r}^{2}+v_{p}^{2}}$ $=0.5$, with beam temperature $T_{b}=8.89 \cdot 10^{-3}$ and average energy $\langle E\rangle=0.147$.

Energies are in units of $v_{0}^{2}$. Both beams are localized in the uniformly discretized velocity subdomain and are depicted in Fig. 5. 

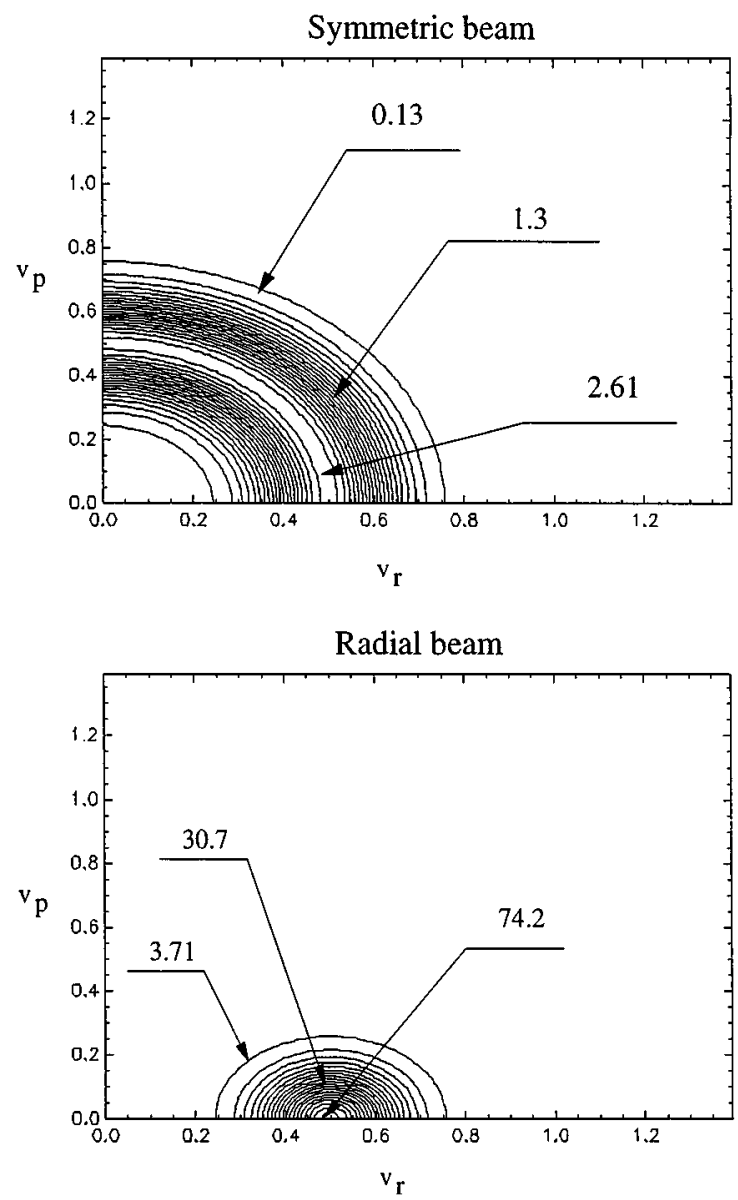

FIG. 5. Test distribution functions employed in the assessment of the energy conservation properties of the discretization. These plots only cover the uniformly discretized region of the domain. Velocity units are arbitrary. Some reference values of the distribution function at selected contours are indicated; contours are equally spaced.

\subsection{Performance of the New Discretization Concept}

To test the success in improving the cancellation of the energy moment of the FokkerPlanck collision operator, the error $\Delta_{E}$ will be calculated and compared for three different discretization schemes:

1. The energy-conservative discretization developed here, with $H$ obtained from its non-linear definition (Eq. (46)). This scheme will be identified by NLS (non-linear solver).

2. The energy-conservative discretization with $H$ obtained from its linear definion (Eq. (3)). This scheme will be identified by LS (linear solver). This case is introduced to address the importance of solving the non-linear problem for $H$ for adequate energy conservation, instead of the more convenient linear approach.

3. A standard particle-conservative, second order discretization of the Fokker-Planck collision operator. This discretization is based upon the particle-conservative discretization in Eq. (34), but using the standard form of the Fokker-Planck flux (Eq. (2)),

$$
\mathbf{J}_{F P}=f \frac{\partial H(f)}{\partial \mathbf{v}}-\frac{1}{2} \frac{\partial}{\partial \mathbf{v}} \cdot\left(\frac{\partial^{2} G(f)}{\partial \mathbf{v} \partial \mathbf{v}} f\right)
$$


In cylindrical coordinates, the Fokker-Planck flux components $\mathbf{J}_{F P}=R \mathbf{u}_{p}+Z \mathbf{u}_{r}$ are, specifically,

$$
\begin{aligned}
& Z=f \frac{\partial H}{\partial v_{r}}-\frac{\partial^{2} G}{\partial v_{r}^{2}} \frac{\partial f}{\partial v_{r}}-\frac{\partial^{2} G}{\partial v_{r} \partial v_{p}} \frac{\partial f}{\partial v_{p}} \\
& R=f \frac{\partial H}{\partial v_{p}}-\frac{\partial^{2} G}{\partial v_{p}^{2}} \frac{\partial f}{\partial v_{p}}-\frac{\partial^{2} G}{\partial v_{r} \partial v_{p}} \frac{\partial f}{\partial v_{r}} .
\end{aligned}
$$

All the derivatives are discretized with centered finite differences in the stencil depicted in Fig. 2. This scheme will be identified by NEC (non-energy-conservative).

Note that no time integration is involved in the calculation of $\Delta_{E}$, which is simply the error introduced in the numerical integration of the energy moment of the Fokker-Planck collision operator. The issue of the propagation of this error in time will be addressed in Ref. [3], where the development of an energy conservative solver, based upon the present difference scheme, is discussed. Suffice it to say here that, as shown in the same reference, $\Delta_{E} \sim 0$ is condition sine-qua-non to construct an energy-conservative time advancing algorithm. This justifies the adequacy of $\Delta_{E}$ as a figure of merit to address the performance of the proposed discretization against other alternatives.

The behavior of the error in the energy moment $\Delta_{E}$ (defined in Eq. (51)) for these three schemes, in terms of $v_{\text {limit }}$ and for different mesh refinements (represented by the number of mesh points, $N=2^{n} \times 2^{n}$ ) is given in Fig. 6. Several important comments are in order from this plot:

- In all curves, the leftmost datapoint $\left(v_{\text {limit }}=\sqrt{2}\right)$ corresponds to the case when there is no geometric mesh region, and only the uniform mesh region is considered. To present a fair comparison of the improvement in the cancellation of the energy moment with $v_{\text {limit }}$, the spacing in the uniform mesh has been kept constant in all datapoints belonging to a same curve. With this in mind, the decreasing trend of the energy moment error $\Delta_{E}$ with $v_{\text {limit }}$ has a clear interpretation, namely, that introducing a geometric mesh to complement the uniform mesh region has a beneficial effect in the energy conservation properties of the NLS scheme in all scenarios, but has little or no effect in the LS and NEC schemes. This is expected, as the geometric region only benefits when the cancellation of the energy moment within the domain is ensured.

- The relative performance of the three different schemes does not depend on the number of mesh points, as evidenced by the fact that the plots for $n=5$ (Figs. 6a, 6c) and $n=7$ (Figs. 6b, 6d) are essentially identical (but for the absolute magnitude of the error).

- There is, however, a strong dependence on the test case (radial/symmetric) and the limit of the velocity domain $v_{\text {limit }}$. Focusing on the radial beam test case (Figs. 6c, 6d), both the NLS and LS schemes clearly outperform the NEC scheme, for any $v_{\text {limit }}$, by at least an order of magnitude. Note that, while the NEC scheme yields a flat error profile with $v_{\text {limit }}$, both the NLS and LS schemes show rapidly decreasing trends in the energy error. However, while the NLS keeps a monotonic exponentially decreasing trend, the energy error in the LS scheme ends up saturating_and even increasing-after a certain $v_{\text {limit }}$ threshold.

- The saturation of the error in the LS scheme, which is mild in the radial beam test case, is deleterious for the symmetric beam (Figs. 6a, 6b). There, both the NEC and the LS 

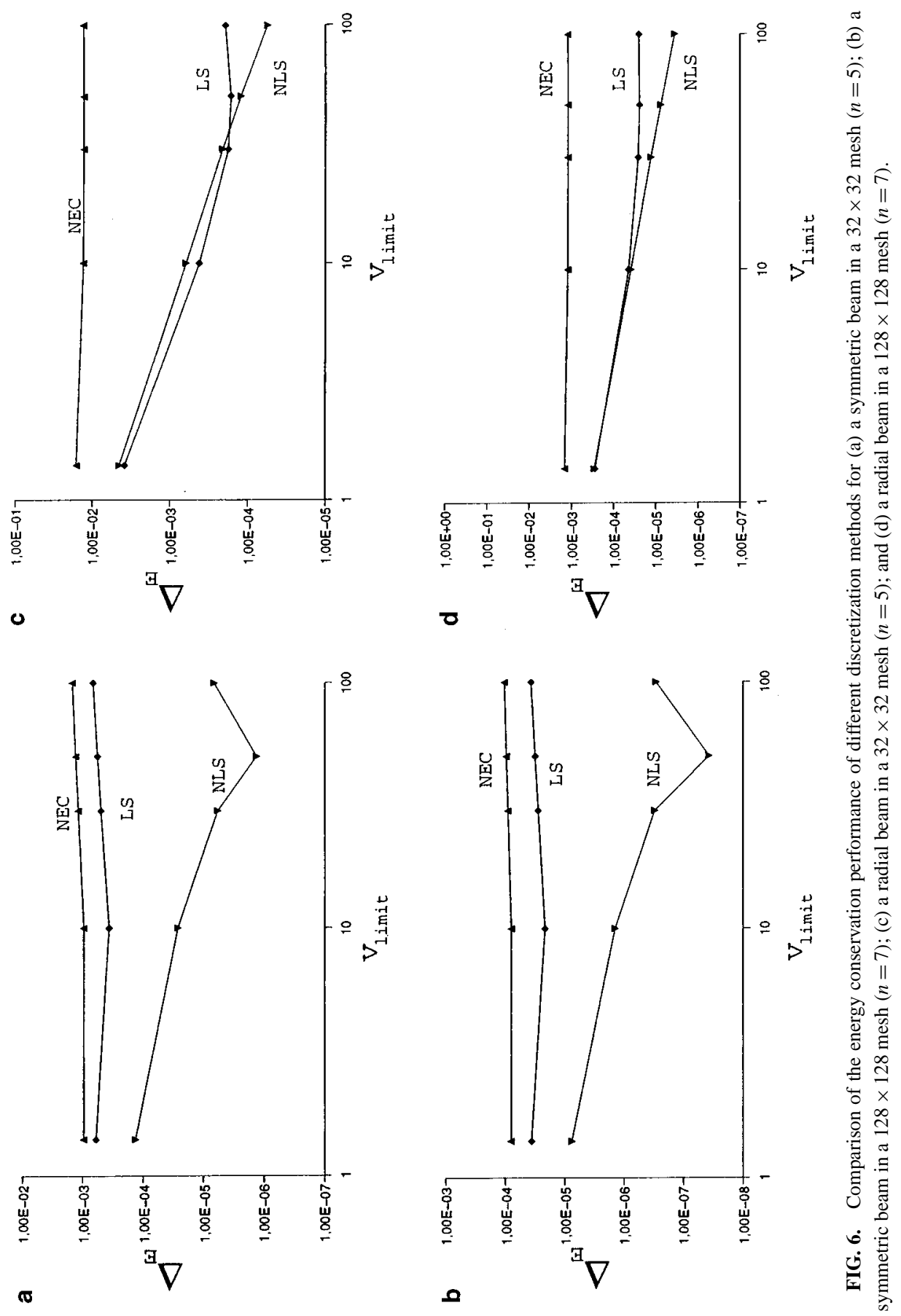
shemes render a flat energy error profile, and only the NLS scheme shows a decreasing trend of the energy error with $v_{\text {limit }}$, although eventually finds a minimum and starts increasing.

These trends clearly indicate two points: first, the difference scheme proposed in this paper succeeds in canceling the energy moment within the domain, as evidenced by the decreasing trend of $\Delta_{E}$ with $v_{\text {limit }}$; otherwise, the expected trend would be flat, as in the NEC scheme. Second, it is essential to solve the non-linear constitutive equation for the $H$ Rosenbluth potential in order to take full advantage of the energy-conservative properties of the difference scheme, as evidenced by the striking differences in performance between the NLS and the LS schemes.

\subsection{Scaling of $\Delta_{E}$}

Although theoretical scaling laws have been derived in Section 4 for three different meshes, namely, uniform mesh, geometric mesh, and a combined uniform-geometric mesh (the latter being the more advantageous in terms of flexibility, resolution, and scaling), only the latter will be considered in this section, for several reasons. First, the flexibility of the combined mesh allows posing extreme cases, both in the number of mesh points and in velocity domain size, without compromising the numerical resolution of the distribution function. Clearly, if pure geometric or uniform meshes were employed, the combination of small $N$ with large velocity domains would result in a lack of resolution of the distribution function that would obscure the scaling of $\Delta_{E}$ (i.e., it would be difficult to assess if the difference in the energy error were due to the scaling law or to lack of resolution). Second, the combined mesh can always be tailored to provide a uniform mesh in the region where the distribution function is located, thus ensuring sufficient resolution. Finally, the combined mesh exhibits the main features of both the uniform and the geometric cases; thus, testing the understanding of the combined mesh essentially tests also the understanding of the pure geometric and uniform mesh cases. For the following numerical experiments, the same velocity domain, discretization mesh, and test cases defined above are used.

5.2.1. Scaling of $\Delta_{E}$ with $v_{\text {limit. }}$ The evolution of $\Delta_{E}$ with $v_{\text {limit }}$ for various mesh refinements is presented in Fig. 7, for both the symmetric beam (Fig. 7a) and the radial beam (Fig. 7b) cases. Note that the behavior of $\Delta_{E}$ in terms of $v_{\text {limit }}$ for the radial and symmetric beams is remarkably different: while in the radial beam case the scaling is monotonic and clearly follows the $1 / v_{\text {limit }}$ prediction, the symmetric beam case is more complex, presenting
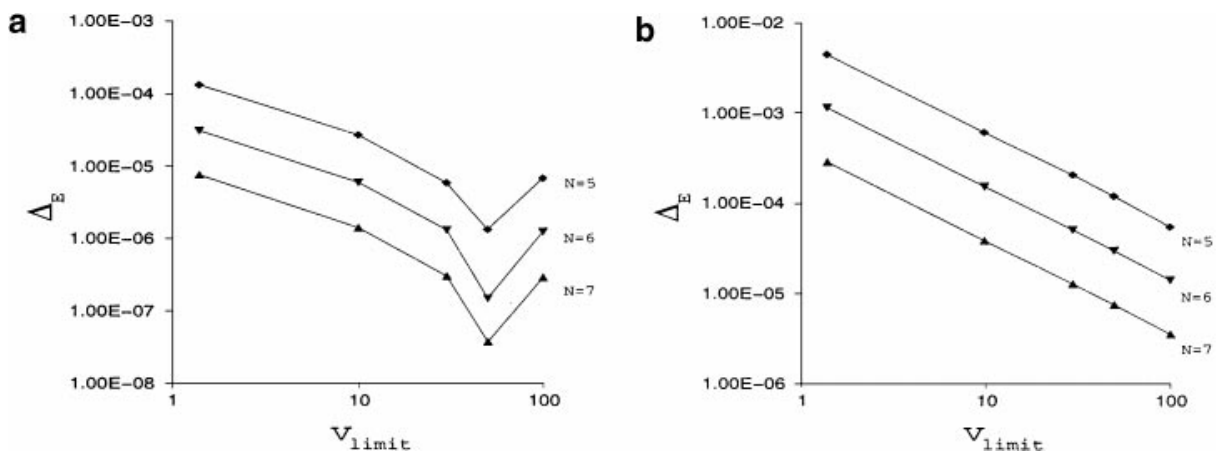

FIG. 7. Scaling of $\Delta_{E}$ with $v_{\text {limit }}$ for various mesh refinements in (a) the symmetric beam test case; (b) the radial beam test case. 

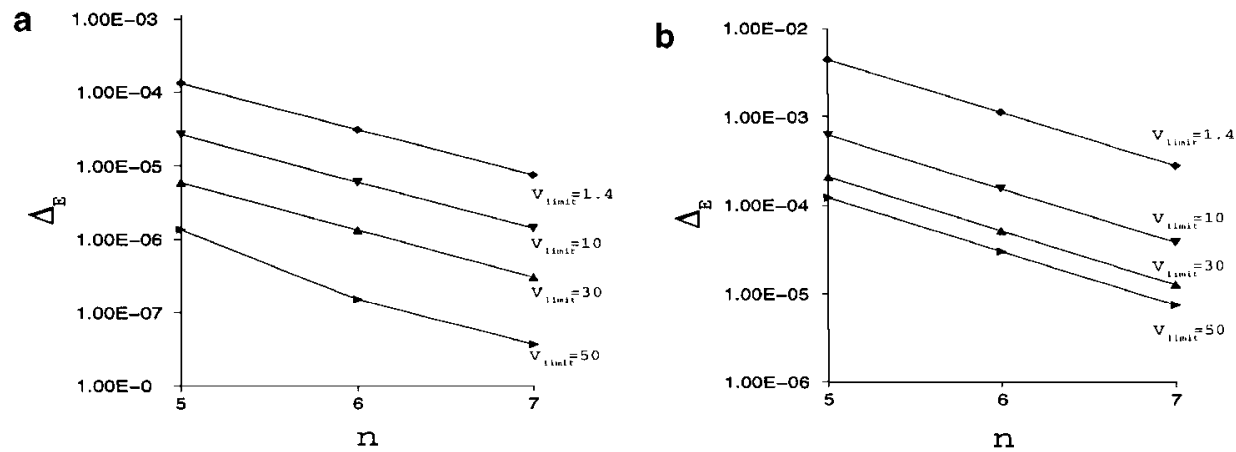

FIG. 8. Scaling of $\Delta_{E}$ with the number of mesh points $N=2^{n} \times 2^{n}$ for different values of $v_{\text {limit }}$ in (a) the symmetric beam test case; (b) the radial beam test case.

four different regions:

- $v_{\text {limit }} \in(1.4,10): \sim 1 / v_{\text {limit }}$ scaling.

- $v_{\text {limit }} \in(10,30)$ : transition region.

- $v_{\text {limit }} \in(30,50): \sim 1 / v_{\text {limit }}^{2}$ scaling.

- $v_{\text {limit }}>50: \sim v_{\text {limit }}$ scaling.

The behavior of the energy error $\Delta_{E}$ in both the symmetric and radial beams is essentially consistent with the scaling analysis presented in Section 4 (see Table I) for the combined uniform-geometric meshes. The differences in the behavior of $\Delta_{E}$ between the symmetric beam and the radial beam are probably due to differences in the magnitude of the leading order term in the energy error: while it is large in the radial beam, dominating the scaling, it is small in the symmetric beam, thus allowing higher order terms of the scaling to surface.

5.2.2. Scaling of $\Delta_{E}$ with the number of mesh points $N$. The evolution of $\Delta_{E}$ with the number of mesh points $N$ for various domain limits is depicted in Fig. 8, for both the symmetric (Fig. 8a) and radial (Fig. 8b) beams. All trends follow the $1 / N$ scaling, as predicted in Table I.

\section{CONCLUSIONS}

The present work has successfully dealt with the task of developing a systematic discretization method of the Fokker-Planck collision operator in multidimensional geometries that preserves particles exactly, and improves energy conservation with respect to previously available approaches. The discretization method can be applied to both uniform meshes and non-uniform meshes.

The development of such a discretization method scheme has been possible by a theoretical reformulation of the Fokker-Planck collision operator into the tensor Fokker-Planck formalism, which yields the symmetries of the theoretical system more conveniently for the development of a conservative numerical representation of the problem. In fact, it has been the numerical preservation of these theoretical symmetries that has driven the development of the discretization scheme.

The Fokker-Planck collision operator is non-linear in that its coefficients are integral functions of the solution sought. As it is known from earlier work in 1D Fokker-Planck [2,9], the numerical representation of these coefficients is crucial to achieve energy conservation. 
This research has shown that this is also the case in multidimensional problems. In particular, it has been found that the $H$ Rosenbluth potential has to be obtained numerically from the discretization of a non-linear differential problem, instead of using the simpler linear Poisson differential formulation (Rosenbluth). Both problems are equivalent in the continuum, but their numerical solutions differ by truncation errors.

This research has shown theoretically that the difference scheme developed herein ensures the exact numerical cancellation of the energy moment within the velocity domain, and that errors in the cancellation occur at the outer boundaries. Heuristic scaling laws for this error in terms of the number of mesh points $N$ and the velocity domain limit $v_{\text {limit }}$ have been derived and validated numerically.

Finally, it should be stressed that, although the development of an energy-conservative discretization method is a necessary condition to develop an energy-conservative solver, it is not sufficient. Thus, this step has to be combined with the appropriate time discretization scheme to construct an energy-conservative Fokker-Planck solver. This task has been undertaken by the authors in Ref. [3].

\section{APPENDIX A}

\section{Particle Conservative Discretization in Velocity Space}

Nine different regions are identified in the velocity domain (inner domain, four boundaries, and four corner points) that will render as many different forms of the volume and surface elements in velocity space. The specialization of Eq. (34) for each of these nine cases is discussed next.

Discretization of $(\partial / \partial \mathbf{v}) \cdot \mathbf{J}_{F P}$ in the inner domain $\left(i \neq 1 ; j \neq 1 ; i \neq N_{r} ; j \neq N_{p}\right)$. The volume element associated to the node $(i, j)$ in cylindrical space is a hollow cylinder with height $\Delta v_{r, i}$, mean radius $v_{p, j}$, and thickness $\Delta v_{p, j}$. Then, the corresponding volume and surface elements for the node $(i, j)$ are

$$
\begin{aligned}
\Delta S_{i+\frac{1}{2}, j} & =\Delta S_{i-\frac{1}{2}, j}=2 \pi v_{p, j} \Delta v_{p, j} \\
\Delta S_{i, j+\frac{1}{2}} & =2 \pi v_{p, j+\frac{1}{2}} \Delta v_{r, i} \\
\Delta S_{i, j-\frac{1}{2}} & =2 \pi v_{p, j-\frac{1}{2}} \Delta v_{r, i} \\
\Delta \Omega_{i, j} & =2 \pi v_{p, j} \Delta v_{p, j} \Delta v_{r, i} .
\end{aligned}
$$

Introducing these results in Eq. (34), the following discretized equation results,

$$
\left[\frac{\partial}{\partial \mathbf{v}} \cdot \mathbf{J}_{F P}\right]_{i, j}=\frac{v_{p, j+\frac{1}{2}} R_{i, j+\frac{1}{2}}-v_{p, j-\frac{1}{2}} R_{i, j-\frac{1}{2}}}{v_{p, j} \Delta v_{p, j}}+\frac{Z_{i+\frac{1}{2}, j}-Z_{i-\frac{1}{2}, j}}{\Delta v_{r, i}}
$$

which is exactly the same result that would have been obtained by discretizing directly the differential expression of the divergence in Eq. (31).

Discretization of $(\partial / \partial \mathbf{v}) \cdot \mathbf{J}_{F P}$ at the $v_{p}=0$ boundary $\left(i \neq 1 ; i \neq N_{r} ; j=1\right)$. The volume corresponding to this velocity node is a solid cylinder of radius $\Delta v_{p, 1} / 2$ and height $\Delta v_{r, i}$. Also, because the control volume corresponding to this node cannot extend further that the $v_{p}=0$ axis, the relevant component of the flux is not $R_{i, 1 / 2}$ at that boundary (the node $j=\frac{1}{2}$ 
does not exist), but $R_{i, 1}$, which is zero for particle conservation. The volume and surface elements corresponding to this volume are

$$
\begin{aligned}
\Delta S_{i+\frac{1}{2}, 1} & =\Delta s_{i-\frac{1}{2}, 1}=\pi\left(\frac{\Delta v_{p, 1}}{2}\right)^{2} \\
\Delta S_{i, \frac{3}{2}} & =2 \pi\left(\frac{\Delta v_{p, 1}}{2}\right) \Delta v_{r, i} \\
\Delta \Omega_{i, 1} & =\pi\left(\frac{\Delta v_{p, 1}}{2}\right)^{2} \Delta v_{r, i} .
\end{aligned}
$$

Then, the discretized divergence operator is, for this case,

$$
\left[\frac{\partial}{\partial \mathbf{v}} \cdot \mathbf{J}_{F P}\right]_{i, j=1}=\frac{4 R_{i, \frac{3}{2}}}{\Delta v_{p, 1}}+\frac{Z_{i+\frac{1}{2}, 1}-Z_{i-\frac{1}{2}, 1}}{\Delta v_{r, i}} .
$$

Note that the singularity present in the differential form of the divergence operator in Eq. (31) at $v_{p}=0(j=1)$ has been resolved in Eq. (57), obtained from the integral formulation. The same result, however, can be obtained from the differential formulation, by realizing that $R \rightarrow 0$ when $v_{p} \rightarrow 0$ by conservation of particles (i.e., the flux normal to the boundary is zero), and hence

$$
\left.\frac{1}{v_{p}} \frac{\partial}{\partial v_{p}}\left(v_{p} R\right)\right|_{v_{p} \rightarrow 0}=\left[\frac{\partial R}{\partial v_{p}}+\frac{R}{v_{p}}\right]_{v_{p} \rightarrow 0}=\left.2 \frac{\partial R}{\partial v_{p}}\right|_{v_{p} \rightarrow 0}
$$

by L'hospital's rule. This result is discretized at the $(i, j=1)$ node as

$$
\left.2 \frac{\partial R}{\partial v_{p}}\right|_{i, j=1}=2 \frac{R_{i, \frac{3}{2}}-R_{i, 1}}{\Delta v_{p, 1} / 2}=\frac{4 R_{i, \frac{3}{2}}}{\Delta v_{p, 1}}
$$

( $R_{i, 1}=0$ for particle conservation), which is exactly the same result as in Eq. (57). Thus, both approaches are equivalent.

Discretization of $(\partial / \partial \mathbf{v}) \cdot \mathbf{J}_{F P}$ at the $v_{r}=0$ boundary $\left(i=1 ; j \neq 1 ; j \neq N_{p}\right)$. The volume element in this case is a hollow cylinder with height $\Delta v_{r, 1} / 2$, and mean radius and thickness given by $v_{p, j}$ and $\Delta v_{p, j}$, respectively. As in the $j=1$ case, the node $i=\frac{1}{2}$ does not exist, and particle conservation forces $Z_{1, j}=0$. Hence, the volume and surface elements read as

$$
\begin{aligned}
\Delta S_{\frac{3}{2}, j} & =2 \pi v_{p, j} \Delta v_{p, j} \\
\Delta S_{1, j+\frac{1}{2}} & =2 \pi v_{p, j+\frac{1}{2}} \frac{\Delta v_{r, 1}}{2} \\
\Delta S_{1, j-\frac{1}{2}} & =2 \pi v_{p, j-\frac{1}{2}} \frac{\Delta v_{r, 1}}{2} \\
\Delta \Omega_{1, j} & =2 \pi v_{p, j} \Delta v_{p, j} \frac{\Delta v_{r, 1}}{2} .
\end{aligned}
$$

The divergence operator of the Fokker-Planck flux at this boundary reads

$$
\left[\frac{\partial}{\partial \mathbf{v}} \cdot \mathbf{J}_{F P}\right]_{i=1, j}=\frac{v_{p, j+\frac{1}{2}} R_{i, j+\frac{1}{2}}-v_{p, j-\frac{1}{2}} R_{1, j-\frac{1}{2}}}{v_{p, j} \Delta v_{p, j}}+\frac{2 Z_{\frac{3}{2}, j}}{\Delta v_{r, 1}} .
$$


Discretization of $(\partial / \partial \mathbf{v}) \cdot \mathbf{J}_{F P}$ at the $v_{p}=v_{\text {limit }}$ boundary $\left(i \neq 1 ; i \neq N_{r} ; j=N_{p}\right)$. The volume element in this case is a hollow cylinder with height $\Delta v_{r, i}$, and inner radius and thickness given by $v_{p, N_{p}-1 / 2}$ and $\Delta v_{p, N_{p}} / 2$, respectively. As in previous cases, particle conservation is imposed at the boundary by forcing $R_{i, N_{p}}=0$. The volume and surface elements are

$$
\begin{aligned}
\Delta S_{i+\frac{1}{2}, N_{p}} & =\Delta S_{i-\frac{1}{2}, N_{p}}=2 \pi v_{p, N_{p}} \frac{\Delta v_{p, N_{p}}}{2} \\
\Delta S_{i, N_{p}-\frac{1}{2}} & =2 \pi v_{p, N_{p}-\frac{1}{2}} \Delta v_{r, i} \\
\Delta \Omega_{i, N_{p}} & =2 \pi v_{p, N_{p}} \frac{\Delta v_{p, N_{p}}}{2} \Delta v_{r, i} .
\end{aligned}
$$

The discretization of the divergence operator of the Fokker-Planck flux results

$$
\left[\frac{\partial}{\partial \mathbf{v}} \cdot \mathbf{J}_{F P}\right]_{i, j=N_{p}}=-\frac{2 v_{p, N_{p}-\frac{1}{2}} R_{i, N_{p}-\frac{1}{2}}}{v_{p, N_{p}} \Delta v_{p, N_{p}}}+\frac{Z_{i+\frac{1}{2}, N_{p}}-Z_{i-\frac{1}{2}, N_{p}}}{\Delta v_{r, i}} .
$$

Discretization of $(\partial / \partial \mathbf{v}) \cdot \mathbf{J}_{F P}$ at the $v_{r}=v_{\text {limit }}$ boundary $\left(i=N_{r} ; j \neq 1 ; j \neq N_{p}\right)$. The volume element associated is a hollow cylinder with height $\Delta v_{r, N_{r}} / 2$, mean radius $v_{p, j}$, and thickness $\Delta v_{p, j}$. Particle conservation requires $Z_{N_{r}, j}=0$. The volume and relevant surface elements are

$$
\begin{aligned}
\Delta S_{N_{r}-\frac{1}{2}, j} & =2 \pi v_{p, j} \Delta v_{p, j} \\
\Delta S_{N_{r}, j+\frac{1}{2}} & =2 \pi v_{p, j+\frac{1}{2}} \frac{\Delta v_{r, N_{r}}}{2} \\
\Delta S_{N_{r}, j-\frac{1}{2}} & =2 \pi v_{p, j-\frac{1}{2}} \frac{\Delta v_{r, N_{r}}}{2} \\
\Delta \Omega_{N_{r, j}} & =2 \pi v_{p, j} \Delta v_{p, j} \frac{\Delta v_{r, N_{r}}}{2} .
\end{aligned}
$$

The discretized divergence operator reads as

$$
\left[\frac{\partial}{\partial \mathbf{v}} \cdot \mathbf{J}_{F P}\right]_{i=N_{r}, j}=\frac{v_{p, j+\frac{1}{2}} R_{N_{r}, j+\frac{1}{2}}-v_{p, j-\frac{1}{2}} R_{N_{r}, j-\frac{1}{2}}}{v_{p, j} \Delta v_{p, j}}-\frac{2 Z_{N_{r}-\frac{1}{2}, j}}{\Delta v_{r, N_{r}}} .
$$

Discretization of $(\partial / \partial \mathbf{v}) \cdot \mathbf{J}_{F P}$ at the $v_{r}=0, v_{p}=0$ corner point $(i=1, j=1)$. The volume element is a solid cylinder with radius $\Delta v_{p, 1} / 2$ and height $\Delta v_{r, 1} / 2$, with outer surfaces and volume given by

$$
\begin{aligned}
& \Delta S_{\frac{3}{2}, 1}=\pi\left(\frac{\Delta v_{p, 1}}{2}\right)^{2} \\
& \Delta S_{1, \frac{3}{2}}=2 \pi \frac{\Delta v_{p, 1}}{2} \frac{\Delta v_{r, 1}}{2} \\
& \Delta \Omega_{1,1}=\pi\left(\frac{\Delta v_{p, 1}}{2}\right)^{2} \frac{\Delta v_{r, 1}}{2} .
\end{aligned}
$$

The discretized equation for the divergence of the Fokker-Planck flux is

$$
\left[\frac{\partial}{\partial \mathbf{v}} \cdot \mathbf{J}_{F P}\right]_{i=1, j=1}=\frac{4 R_{1, \frac{3}{2}}}{\Delta v_{p, 1}}+\frac{2 Z_{\frac{3}{2}, 1}}{\Delta v_{r, 1}}
$$


where the components of the flux that cross the boundaries have been set to zero to conserve particles.

Discretization of $(\partial / \partial \mathbf{v}) \cdot \mathbf{J}_{F P}$ at the $v_{r}=0, v_{p}=v_{\text {limit }}$ corner point $\left(i=1, j=N_{p}\right)$. The volume element is a hollow cylinder with inner radius $v_{p, N_{r}-1 / 2}$, thickness $\Delta v_{p, N_{r}} / 2$, and height $\Delta v_{r, 1} / 2$, and outer surfaces and volume given by

$$
\begin{aligned}
\Delta S_{\frac{3}{2}, N_{p}} & =2 \pi v_{p, N_{p}} \frac{\Delta v_{p, N_{p}}}{2} \\
\Delta S_{1, N_{p}-\frac{1}{2}} & =2 \pi v_{p, N_{p}-\frac{1}{2}} \frac{\Delta v_{r, 1}}{2} \\
\Delta \Omega_{1, N_{r}} & =2 \pi v_{p, N_{p}} \frac{\Delta v_{p, N_{p}}}{2} \frac{\Delta v_{r, 1}}{2}
\end{aligned}
$$

and the discretized equation for the divergence of the Fokker-Planck flux is

$$
\left[\frac{\partial}{\partial \mathbf{v}} \cdot \mathbf{J}_{F P}\right]_{i=1, j=N_{p}}=-\frac{2 v_{p, N_{p}-\frac{1}{2}} R_{1, N_{p}-\frac{1}{2}}}{v_{p, N_{p}} \Delta v_{p, N_{p}}}+\frac{2 Z_{\frac{3}{2}, N_{p}}}{\Delta v_{r, N_{p}}} .
$$

Particle conservative boundary conditions have been imposed as usual.

Discretization of $(\partial / \partial \mathbf{v}) \cdot \mathbf{J}_{F P}$ at the $v_{r}=v_{\text {limit }}, v_{p}=0$ corner point $\left(i=N_{r}, j=1\right)$. The volume corresponding to this velocity node is a solid cylinder of radius $\Delta v_{p, 1} / 2$ and height $\Delta v_{r, N_{r}} / 2$. Hence

$$
\begin{aligned}
\Delta S_{N_{r}-\frac{1}{2}, 1} & =\pi\left(\frac{\Delta v_{p, 1}}{2}\right)^{2} \\
\Delta S_{N_{r}, \frac{3}{2}} & =2 \pi\left(\frac{\Delta v_{p, 1}}{2}\right) \frac{\Delta v_{r, N_{r}}}{2} \\
\Delta \Omega_{N_{r}, 1} & =\pi\left(\frac{\Delta v_{p, 1}}{2}\right)^{2} \frac{\Delta v_{r, N_{r}}}{2} .
\end{aligned}
$$

The discretized divergence operator reads, after imposing the boundary conditions,

$$
\left[\frac{\partial}{\partial \mathbf{v}} \cdot \mathbf{J}_{F P}\right]_{i=N_{r}, j=1}=\frac{4 R_{N_{r}, \frac{3}{2}}}{\Delta v_{p, 1}}-\frac{2 Z_{N_{r}-\frac{1}{2}, 1}}{\Delta v_{r, N_{r}}} .
$$

Discretization of $(\partial / \partial \mathbf{v}) \cdot \mathbf{J}_{F P}$ at the $v_{r}=v_{\text {limit }}, v_{p}=v_{\text {limit }}$ corner point $\left(i=N_{r}, j=N_{p}\right)$. The volume corresponding to this velocity node is a hollow cylinder of inner radius $v_{p, N_{p}-1 / 2}$, thickness $\Delta v_{p, N_{p}} / 2$, and height $\Delta v_{r, N_{r}} / 2$. Hence

$$
\begin{aligned}
\Delta S_{N_{r}-\frac{1}{2}, N_{p}} & =2 \pi v_{p, N_{p}} \frac{\Delta v_{p, N_{p}}}{2} \\
\Delta S_{N_{r}, N_{p}-\frac{1}{2}} & =2 \pi v_{p, N_{p}-\frac{1}{2}} \frac{\Delta v_{r, N_{r}}}{2} \\
\Delta \Omega_{N_{r}, N_{p}} & =2 \pi v_{p, N_{p}} \frac{\Delta v_{p, N_{p}}}{2} \frac{\Delta v_{r, N_{r}}}{2} .
\end{aligned}
$$


The discretized divergence yields, after imposing the boundary conditions,

$$
\left[\frac{\partial}{\partial \mathbf{v}} \cdot \mathbf{J}_{F P}\right]_{i=N_{r}, N_{p}}=-\frac{2 v_{p, N_{p}-\frac{1}{2}} R_{N_{r}, N_{p}-\frac{1}{2}}}{v_{p, N_{p}} \Delta v_{p, N_{p}}}-\frac{2 Z_{N_{r}-\frac{1}{2}, N_{p}}}{\Delta v_{r, N_{r}}} .
$$

\section{APPENDIX B}

\section{Integration of Energy Error at the Boundaries}

The error at the boundaries stemming from a second order discretization reads, according to Eq. (52),

$$
\Delta_{1} \sim \int_{\partial \Omega} d \mathbf{S} \cdot \mathbf{v}\left(\left[\Delta v_{r} \frac{\partial}{\partial v_{r}}+\Delta v_{p} \frac{\partial}{\partial v_{p}}\right]^{(2)} \frac{\partial H_{0}}{\partial \mathbf{v}}\right) \cdot \frac{\partial H_{0}}{\partial \mathbf{v}}+O\left(\Delta v^{4}\right) .
$$

This integral is restricted to the outer boundaries of the velocity domain considered in Subsection 3.1, defined by $\partial \Omega_{r}=\left\{v_{r} \in\left[0, v_{\text {limit }}\right], v_{p}=v_{\text {limit }}\right\}$ and $\partial \Omega_{p}=\left\{v_{p} \in\left[0, v_{\text {limit }}\right], v_{r}=\right.$ $\left.v_{\text {limit }}\right\}$. Expanding the term in square brackets in Eq. (67),

$$
\begin{aligned}
{\left[\Delta v_{r} \frac{\partial}{\partial v_{r}}+\Delta v_{p} \frac{\partial}{\partial v_{p}}\right]^{(2)} \frac{\partial H_{0}}{\partial \mathbf{v}} } & =\left[\left(\Delta v_{r}\right)^{2} \frac{\partial^{2}}{\partial v_{r}^{2}}+\left(\Delta v_{p}\right)^{2} \frac{\partial^{2}}{\partial v_{p}^{2}}+2 \Delta v_{p} \Delta v_{r} \frac{\partial^{2}}{\partial v_{r} \partial v_{p}}\right] \frac{\partial H_{0}}{\partial \mathbf{v}} \\
& =\frac{\partial}{\partial \mathbf{v}}\left[\left(\Delta v_{r}\right)^{2} \frac{\partial^{2} H_{0}}{\partial v_{r}^{2}}+\left(\Delta v_{p}\right)^{2} \frac{\partial^{2} H_{0}}{\partial v_{p}^{2}}+2 \Delta v_{p} \Delta v_{r} \frac{\partial^{2} H_{0}}{\partial v_{r} \partial v_{p}}\right] .
\end{aligned}
$$

The last step can be done because angular symmetry allows $\partial / \partial v_{r}, \partial / \partial v_{p}$, and $\partial / \partial \mathbf{v}$ to commute, and it is assumed that the variations of the increments with $v_{r}, v_{p}$ are negligible compared to the variations of $H_{0}$. If $v_{\text {limit }} \gg v_{0}$, then $H_{0} \sim K\left(v_{0} / v\right)=K v_{0} / \sqrt{v_{r}^{2}+v_{p}^{2}}$, and

$$
\begin{aligned}
\frac{\partial H_{0}}{\partial v_{r}} & =-K v_{0} \frac{v_{r}}{v^{3}} \\
\frac{\partial H_{0}}{\partial v_{p}} & =-K v_{0} \frac{v_{p}}{v^{3}} \\
\frac{\partial^{2} H_{0}}{\partial v_{r}^{2}} & =-K v_{0} \frac{1-3\left(v_{r}^{2} / v^{2}\right)}{v^{3}} \\
\frac{\partial^{2} H_{0}}{\partial v_{p}^{2}} & =-K v_{0} \frac{1-3\left(v_{p}^{2} / v^{2}\right)}{v^{3}} \\
\frac{\partial^{2} H_{0}}{\partial v_{p} \partial v_{r}} & =K v_{0} \frac{3 v_{r} v_{p}}{v^{5}} .
\end{aligned}
$$

With these results, and dropping the $K v_{0}$ constant, we find

$$
\begin{gathered}
\left(\Delta v_{r}\right)^{2} \frac{\partial^{2} H_{0}}{\partial v_{r}^{2}}+\left(\Delta v_{p}\right)^{2} \frac{\partial^{2} H_{0}}{\partial v_{p}^{2}}+2 \Delta v_{p} \Delta v_{r} \frac{\partial^{2} H_{0}}{\partial v_{r} \partial v_{p}} \\
=\frac{\left(\Delta v_{r}\right)^{2}+\left(\Delta v_{p}\right)^{2}}{v^{3}}-\frac{3\left(v_{r} \Delta v_{r}+v_{p} \Delta v_{p}\right)^{2}}{v^{5}}
\end{gathered}
$$


which, introduced in the energy error integral, yields

$$
\begin{aligned}
\Delta_{1} \sim & \int_{\partial \Omega} d \mathbf{S} \cdot \mathbf{v}\left[\frac{\partial H_{0}}{\partial v_{r}} \frac{\partial}{\partial v_{r}}\left(\frac{\left(\Delta v_{r}\right)^{2}+\left(\Delta v_{p}\right)^{2}}{v^{3}}-\frac{3\left(v_{r} \Delta v_{r}+v_{p} \Delta v_{p}\right)^{2}}{v^{5}}\right)\right. \\
& \left.+\frac{\partial H_{0}}{\partial v_{p}} \frac{\partial}{\partial v_{p}}\left(\frac{\left(\Delta v_{r}\right)^{2}+\left(\Delta v_{p}\right)^{2}}{v^{3}}-\frac{3\left(v_{r} \Delta v_{r}+v_{p} \Delta v_{p}\right)^{2}}{v^{5}}\right)\right]+O\left(\Delta v^{4}\right) .
\end{aligned}
$$

Carrying the derivatives through and grouping terms, the following results,

$$
\begin{aligned}
\Delta_{1} \sim & \int_{\partial \Omega} d \mathbf{S} \cdot \mathbf{v}\left(\frac{\left(\Delta v_{r}\right)^{2}+\left(\Delta v_{p}\right)^{2}}{v^{6}}-\frac{3\left(v_{r} \Delta v_{r}+v_{p} \Delta v_{p}\right)^{2}}{v^{8}}\right) \\
= & 2 \pi v_{\text {limit }} \int_{0}^{v_{\text {limit }}} d v_{r} v_{r}\left(\frac{\left(\Delta v_{r}\right)^{2}+\left(\Delta v_{p}\right)_{v_{p}=v_{\text {limit }}}^{2}}{v^{6}}-\frac{3\left(v_{r} \Delta v_{r}+v_{\text {limit }}\left(\Delta v_{p}\right)_{v_{p}=v_{\text {limit }}}\right)^{2}}{v^{8}}\right) \\
& +2 \pi \int_{0}^{v_{\text {limit }}} d v_{p} v_{p}^{2}\left(\frac{\left(\Delta v_{r}\right)_{v_{r}=v_{\text {limit }}}^{2}+\left(\Delta v_{p}\right)^{2}}{v^{6}}-\frac{3\left(v_{\text {limit }}\left(\Delta v_{r}\right)_{v_{r}=v_{\text {limit }}}+v_{p} \Delta v_{p}\right)^{2}}{v^{8}}\right) \\
& +O\left(\Delta v^{4}\right) .
\end{aligned}
$$

Since the value of $v$ does not change drastically at the boundaries $\left(v_{\text {limit }} \leq v \leq v_{\text {limit }} \sqrt{2}\right)$, we assume $v \sim v_{\text {limit }}$ (hence, coming out of the integral), and $\Delta_{1}$ simplifies to

$$
\begin{aligned}
& \Delta_{1} \sim-\frac{\left(\Delta v_{p}\right)_{v_{p}=v_{\text {limit }}}^{2}+\frac{2}{3}\left(\Delta v_{r}\right)_{v_{r}=v_{\text {limit }}}^{2}}{v_{\text {limit }}^{3}}+\frac{\int_{0}^{v_{\text {limit }}} d v_{r} v_{r}\left(\Delta v_{r}\right)^{2}}{v_{\text {limit }}^{5}}+\frac{\int_{0}^{v_{\text {limit }}} d v_{p} v_{p}^{2}\left(\Delta v_{p}\right)^{2}}{v_{\text {limit }}^{6}} \\
& -6\left[\frac{\left(\Delta v_{p}\right)_{v_{p}=v_{\text {limit }}} \int_{0}^{v_{\text {limit }}} d v_{r} v_{r}^{2} \Delta v_{r}}{v_{\text {limit }}^{6}}+\frac{\left(\Delta v_{r}\right)_{v_{r}=v_{\text {limit }}} \int_{0}^{v_{\text {limit }}} d v_{p} v_{p}^{3} \Delta v_{p}}{v_{\text {limit }}^{7}}\right] \\
& -3\left[\frac{\int_{0}^{v_{\text {limit }}} d v_{r} v_{r}^{3}\left(\Delta v_{r}\right)^{2}}{v_{\text {limit }}^{7}}+\frac{\int_{0}^{v_{\text {limit }}} d v_{p} v_{p}^{4}\left(\Delta v_{p}\right)^{2}}{v_{\text {limit }}^{8}}\right]+O\left(\Delta v^{4}\right),
\end{aligned}
$$

where the $2 \pi$ constant has been dropped for simplicity. To perform the remaining integrals, information about the details of the discretization of the velocity domain is needed. Three possibilities are considered at this point: uniform mesh, geometric mesh, and a combined uniform/geometric mesh.

B.1. Uniform mesh. In a uniform mesh, the velocity increments are constant and the integrals in Eq. (68) are rendered trivial. Recovering the $K v_{0}$ constant, the following expression results:

$$
\Delta_{1} \sim-\frac{K v_{0}\left(\Delta v_{r}^{2}+1.4 \Delta v_{p}^{2}+3.8 \Delta v_{r} \Delta v_{p}\right)}{v_{\text {limit }}^{3}} .
$$

B.2. Geometric mesh. In a geometric mesh defined by the ratio $\xi$ (which will be assumed equal in both $v_{r}$ and $v_{p}$ directions for simplicity), the velocity increments satisfy

$$
\begin{aligned}
\Delta v_{r, i} & =\xi \Delta v_{r, i-1}=\xi^{i-1} \Delta v_{r, 1} \\
\Delta v_{p, j} & =\xi \Delta v_{p, j-1}=\xi^{j-1} \Delta v_{p, 1}
\end{aligned}
$$


and the radial and perpendicular velocities at the node $(i, j)$ are given by

$$
\begin{gathered}
v_{r, i}=\sum_{n=1}^{i} \Delta v_{r, n}=\frac{\xi^{i}-1}{\xi-1} \Delta v_{r, 1} \\
v_{p, j}=\sum_{n=1}^{j} \Delta v_{p, n}=\frac{\xi^{j}-1}{\xi-1} \Delta v_{p, 1} .
\end{gathered}
$$

At the outer boundaries, $i=N_{r}, j=N_{p}$. Typically, $N_{r} \gg 1, N_{p} \gg 1$. As $\xi>1$, then $\xi^{N_{r}} \gg 1$, $\xi^{N_{p}} \gg 1$ (provided that $\xi>1+1 / N_{r}$ and $\xi>1+1 / N_{p}$ ), and we can write

$$
\begin{aligned}
& v_{\text {limit }} \sim \frac{\xi^{N_{r}} \Delta v_{r, 1}}{\xi-1} \sim \frac{\Delta v_{r, N_{r}}}{\xi-1} \Rightarrow \Delta v_{r, N_{r}}=\left(\Delta v_{r}\right)_{v_{\text {limit }}} \sim(\xi-1) v_{\text {limit }} \\
& v_{\text {limit }} \sim \frac{\xi^{N_{p}} \Delta v_{p, 1}}{\xi-1} \sim \frac{\Delta v_{p, N_{p}}}{\xi-1} \Rightarrow \Delta v_{p, N_{p}}=\left(\Delta v_{p}\right)_{v_{\text {limit }}} \sim(\xi-1) v_{\text {limit }} .
\end{aligned}
$$

With this information, the integrals in Eq. (68) can be calculated as follows. Take, for instance, the first integral, given by $\int_{0}^{v_{\text {limit }}} d v_{r} v_{r}\left(\Delta v_{r}\right)^{2}$, and discretize it so that

$$
\int_{0}^{v_{\text {limit }}} d v_{r} v_{r}\left(\Delta v_{r}\right)^{2} \simeq \sum_{i=1}^{N_{r}-1} v_{r, i}\left(\Delta v_{r, i}\right)^{3}=\frac{\left(\Delta v_{r, 1}\right)^{4}}{\xi^{3}(\xi-1)} \sum_{i=1}^{N_{r}-1}\left(\xi^{4 i}-\xi^{3 i}\right)
$$

Equations (69) and (71) have been used for the last step. Each of the two sums in the previous result is the sum of a geometric series, with ratios given by $\xi^{4}$ and $\xi^{3}$, respectively. Hence, the sum can be performed to find

$$
\int_{0}^{v_{\text {limit }}} d v_{r} v_{r}\left(\Delta v_{r}\right)^{2} \simeq \frac{\left(\Delta v_{r, 1}\right)^{4}}{\xi^{3}(\xi-1)}\left[\frac{\xi^{4 N_{r}}-1}{\xi^{4}-1}-\frac{\xi^{3 N_{r}}-1}{\xi^{3}-1}\right] .
$$

Recalling that $\xi^{N_{r}} \gg 1$, and using Eq. (69) again, the following results:

$$
\int_{0}^{v_{\text {limit }}} d v_{r} v_{r}\left(\Delta v_{r}\right)^{2} \approx \frac{\left(\Delta v_{r, N_{r}}\right)^{3}}{\xi^{3}(\xi-1)}\left[\frac{\xi^{4} \Delta v_{r, N r}}{\xi^{4}-1}-\frac{\xi^{3} \Delta v_{r, 1}}{\xi^{3}-1}\right] .
$$

Neglecting the second term in the right hand side $\left(\Delta v_{r, 1} \ll \Delta v_{r, N_{r}}\right)$, and using Eq. (73), the integral finally yields

$$
\int_{0}^{v_{\text {limit }}} d v_{r} v_{r}\left(\Delta v_{r}\right)^{2} \sim \frac{v_{\text {limit }}^{4}(\xi-1)^{3}}{\xi^{4}-1} .
$$

All the integrals in Eq. (68) can be calculated in a similar way, yielding

$$
\begin{aligned}
\int_{0}^{v_{\text {limit }}} d v_{p} v_{p}^{2}\left(\Delta v_{p}\right)^{2} & \sim \frac{v_{\text {limit }}^{5}(\xi-1)^{3}}{\xi^{5}-1} \\
\int_{0}^{v_{\text {limit }}} d v_{r} v_{r}^{2} \Delta v_{r} & \sim \frac{v_{\text {limit }}^{4}(\xi-1)^{2}}{\xi^{4}-1} \\
\int_{0}^{v_{\text {limit }}} d v_{p} v_{p}^{3} \Delta v_{p} & \sim \frac{v_{\text {limit }}^{5}(\xi-1)^{2}}{\xi^{5}-1}
\end{aligned}
$$




$$
\begin{aligned}
\int_{0}^{v_{\text {limit }}} d v_{r} v_{r}^{3}\left(\Delta v_{r}\right)^{2} & \sim \frac{v_{\text {limit }}^{6}(\xi-1)^{3}}{\xi^{6}-1} \\
\int_{0}^{v_{\text {limit }}} d v_{p} v_{p}^{4}\left(\Delta v_{p}\right)^{2} & \sim \frac{v_{\text {limit }}^{7}(\xi-1)^{3}}{\xi^{7}-1} .
\end{aligned}
$$

Introducing all these integrals in Eq. (68), together with Eqs. (73) and (74), it follows that

$$
\Delta_{1} \sim-\frac{v_{0}(\xi-1)^{2}}{v_{\text {limit }}}\left[\frac{5}{3}+(\xi-1)\left(\frac{5}{\xi^{4}-1}+\frac{5}{\xi^{5}-1}+\frac{3}{\xi^{6}-1}+\frac{3}{\xi^{7}-1}\right)\right] .
$$

B.3. Combined uniform/geometric mesh. In an attempt to provide sufficient accuracy where it is needed (namely, the region where the bulk of $f$ is), the velocity domain may be discretized uniformly there, and with a geometric mesh elsewhere. Also, the outer boundaries may be discretized with a finer mesh to decrease the energy error introduced at the boundary and to improve its scaling.

Since the uniform region is typically very small compared to the whole domain (the most important physics will occur for $v<5 v_{0}$, and $v_{\text {limit }} / v_{0} \gg 1$ ), its contribution to $\Delta_{1}$ at the boundaries can be neglected. The rest of the outer boundaries is in fact discretized with a geometric mesh, and most of the development in the previous section applies.

On the contrary, a finer discretization at the outer boundaries will indeed have a noticeable effect. This is done by decreasing the velocity increment associated with the last nodes in both $v_{r}$ and $v_{p}$ directions; typically, this increment will be set equal to the increment of the uniform mesh, $\Delta v_{u n} \sim 5 v_{0} / \sqrt{N}$. Hence, $\left(\Delta v_{p}\right)_{v_{p}=v_{\text {limit }}}=\left(\Delta v_{r}\right)_{v_{r}=v_{\text {limit }}}=\Delta v_{\text {un }}$. Introducing these increments in Eq. (68), together with the integrals performed for the geometric mesh, it follows that, for the combined mesh,

$$
\Delta_{1} \sim \frac{(\xi-1)^{2} v_{0}}{v_{\text {limit }}}\left[C_{1}(\xi)+C_{2}(\xi) \frac{v_{0}}{(\xi-1) \sqrt{N} v_{\text {limit }}}+C_{3}(\xi)\left(\frac{v_{0}}{(\xi-1) \sqrt{N} v_{\text {limit }}}\right)^{2}\right]
$$

where the coefficients are given by

$$
\begin{aligned}
& C_{1}(\xi)=(\xi-1)\left[\frac{1}{\xi^{4}-1}+\frac{1}{\xi^{5}-1}-\frac{3}{\xi^{6}-1}-\frac{3}{\xi^{7}-1}\right] \approx-\frac{1}{2} \\
& C_{2}(\xi)=-30(\xi-1)\left[\frac{1}{\xi^{4}-1}+\frac{1}{\xi^{5}-1}\right] \approx-13 \\
& C_{3}(\xi)=-\frac{125}{3}
\end{aligned}
$$

The numerical values are the limits when $\xi \rightarrow 1$.

\section{ACKNOWLEDGMENTS}

This work has been developed under OFES funding at Los Alamos National Laboratory. L.C. has been partially funded by a Fellowship of the Spanish Ministry of Education and Culture, and this research is part of his Ph.D. dissertation at the University of Illinois. 


\section{REFERENCES}

1. J. S. Chang and G. Cooper, A practical difference scheme for Fokker-Planck equations, J. Comput. Phys. 6, 1 (1970).

2. E. M. Epperlein, Implicit and conservative difference scheme for the Fokker-Planck equation, J. Comput. Phys. 112, 291 (1994).

3. L. Chacón, D. C. Barnes, D. A. Knoll, and G. H. Miley, An implicit energy-conservative 2D Fokker-Planck algorithm. II. Jacobian-free Newton-Krylov solver, J. Comput. Phys. 157, 654 (2000).

4. M. N. Rosenbluth, W. M. Macdonald, and D. L. Judd, Fokker-Planck equation for an inverse-square force, Phys. Rev. 107, 1 (1957).

5. F. L. Hinton, Collisional transport in plasma, in Handbook of Plasma Physics, edited by M. N. Rosenbluth and R. Z. Sagdeev (North-Holland, Amsterdam, 1984), Vol. 1, p. 147.

6. Y. Saad, Iterative Methods for Sparse Linear Systems (PWS, Boston, 1996).

7. J. D. Jackson, Classical Electrodynamics, 2nd ed. (Wiley, New York, 1975), p. 239.

8. M. Abramowitz and I. A. Stegun (Eds.), Handbook of Mathematical Functions, 9th ed. (Dover, New York, 1972).

9. T. H. Kho, Relaxation of a system of charged particles, Phys. Rev. A 32, 666 (1985). 